H. Saito

Nagoya Math. J.

Vol. 80 (1980), 129-165

\title{
ON A DECOMPOSITION OF SPACES OF CUSP FORMS AND TRACE FORMULA OF HECKE OPERATORS
}

\author{
HIROSHI SAITO
}

\section{Introduction}

For a positive integer $N$, put

$$
\Gamma_{0}(N)=\left\{\left(\begin{array}{ll}
a & b \\
c & d
\end{array}\right) \in S L_{2}(Z) \mid c \equiv 0(\bmod N)\right\} .
$$

For a positive integer $\kappa$ and a Dirichlet character $\psi$ modulo $N$, let $S_{\varepsilon}(N, \psi)$ denote the space of holomorphic cusp forms for $\Gamma_{0}(N)$ of weight $\kappa$ and character $\psi$. For a positive integer $n$ prime to $N$, the Hecke operator $T_{n}$ is defined on $S_{x}(N, \psi)$, and in the case where $k \geq 2$, an explicit formula for the trace $\operatorname{tr} T_{n}$ of $T_{n}$ is known by Eichler [6] and Hijikata [8]. But for higher levels, in particular, when $N$ contains a power of a prime as a factor, this formula is not suitable for numerical computations. It is natural to ask a decomposition of $S_{k}(N, \psi)$ stable under the action of Hecke operators and a formula for $\operatorname{tr} T_{n}$ on each subspace. In fact, when $\psi$ is the trivial character $\psi_{1}$, Yamauchi [18] gave a decomposition of $S_{k}\left(N, \psi_{1}\right)$ and a formula for $\operatorname{tr} T_{n}$ on each subspace by means of the normalizers of $\Gamma_{0}(N)$. In the case where $N=p^{v}$ with a prime $p, S_{k}\left(p^{v}, \psi_{1}\right)$ is divided into two subspaces by this decomposition. When $\nu \geq 2$, in Saito-Yamauchi [11] another decomposition of $S_{s}\left(p^{v}, \psi_{1}\right)$ into four subspaces and the formulas for $\operatorname{tr} T_{n}$ on these subspaces were given by using the normalizer $W=\left(\begin{array}{ll}0 & -1 \\ p^{\nu} & 0\end{array}\right)$ of $\Gamma_{0}\left(p^{\nu}\right)$ and the twisting operator $R_{\varepsilon}$ for $\varepsilon$ the quadratic residue symbol modulo $p$. In this paper, we shall generalize these results. In $\S 1$, we define an operator $U_{x}$ on $S_{\kappa}(N, \psi)$ for a character $\chi$ which satisfies a certain condition. This operator is a generalization of $R_{\epsilon} W R_{\epsilon} W$ in [11]. In a similar way as in [11], we can give a formula for $\operatorname{tr} U_{x} T_{n}$ and also for $\operatorname{tr} U_{x} W T_{n}$ with a normalizer $W$ of $\Gamma_{0}(N)$ when $\psi$ is trivial ( $\S 2$. Th. 2.5. and Th. 2.9.). In $\S 3$, we shall prove a multiplicative property of $U_{x}$. This

Received May 8, 1979. 
property makes it possible to define a decomposition of $S_{c}(N, \psi)$ into subspaces. This decomposition is finer than the one given in [11] even in the case where $N=p^{3}$ and is trivial. The trace of $T_{n}$ on each subspace is given by a linear combination of $\operatorname{tr} U_{x} T_{n}$ and $\operatorname{tr} U_{x} W T_{n}$. In $\S 4$, we give a numerical example for $N=11^{3}, \kappa=2$ and the trivial $\psi$. In this example, we find a congruence between a cusp form associated with a Grössencharacter of $Q(\sqrt{-11})$ and a certain primitive cusp form modulo a prime ideal $\mathfrak{p}$ with the norm 99527. By means of a result of Shimura [16], this prime ideal can be related to the special values of certain $L$-functions of $\boldsymbol{Q}$ and $\boldsymbol{Q}(\sqrt{-11})$. We can observe such a congruence also in the examples of Doi-Yamauchi [3] for $N=7^{3}$ and [11] for $N=11^{3}$. These observations were done under the influence of Doi-Ohta [4] and Doi-Hida [5]. In the Appendix, we give more examples for $N=13^{3}, 19^{3}$ under the condition that $\kappa=2$ and $\psi$ is trivial.

\section{Notation}

The symbols $Z, \boldsymbol{Q}, \boldsymbol{R}$, and $\boldsymbol{C}$ denote respectively the ring of rational integers, the rational number field, the real number field, and the complex number field. For a prime $p, Z_{p}$ and $\boldsymbol{Q}_{p}$ denote the ring of $p$-adic integers and the field of $p$-adic numbers, respectively. For a prime $p, v_{p}$ denotes the additive valuation of $\boldsymbol{Q}_{p}$ normalized as $v_{p}(p)=1$. For an associative ring $S$ with an identity element, we denote by $S^{\times}$the group of all invertible elements of $S$, and by $M_{n}(S)$ the ring of all square matrices of size $n$ with coefficients in $S$. We put $G L_{n}(S)=M_{n}(S)^{\times}$. For subsets $S_{i j}$ of $S, 1 \leq i, j \leq n,\left(S_{i j}\right)$ denotes the subsets $\left\{\left(s_{i j}\right) \in M_{n}(S) \mid s_{i j} \in S_{i j}\right\}$. For a group $G$ and its subgroup $H$, we denote by $\widetilde{H}$ the conjugacy with respect to $H$, i.e., $g_{\widetilde{H}} g^{\prime}$ if and only if $h^{-1} g h=g^{\prime}$ with $h \in H$, and for a subset $X$ of $G$, we denote by $X /_{\widetilde{H}}$ a complete system of representatives of $X$ with respect to $H$. Finally, for a finite dimensional vector space $V$ over $C$ and a linear operator $T$ on $V, \operatorname{tr} T \mid V$ denotes the trace of $T$ on $V$.

\section{§1. The operator $U_{x}$}

Let $\mathscr{S}_{\mathrm{S}}$ denote the complex upper half plane $\{z \in C \mid \operatorname{Im}(z)>0\}$ and $G L_{2}(R)^{+}$ $=\left\{\gamma \in G L_{2}(R) \mid \operatorname{det} \gamma>0\right\}$. Let $\kappa$ be a positive integer. For a complexvalued function $f(z)$ on $\mathscr{S}$ and $\gamma=\left(\begin{array}{ll}a & b \\ c & d\end{array}\right) \in G L_{2}(R)^{+}$, we define a function $f \mid[\gamma]_{\kappa}$ on $\mathfrak{S}$ by 


$$
\left(f \mid[\gamma]_{\kappa}\right)(z)=(\operatorname{det} \gamma)^{\kappa / 2}(c z+d)^{-\kappa} f(\gamma(z)),
$$

where $\gamma(z)=(a z+b) /(c z+d)$ for $z \in \mathfrak{S}$. For a positive integer $N$ and a Dirichlet character $\psi$ modulo $N$ such that $\psi(-1)=(-1)^{x}$, let $G_{x}(N, \psi)$ denote the vector space of holomorphic modular forms $f(z)$ satisfying

$$
f \mid[\gamma]_{c}=\psi(d) f \quad \text { for all } \gamma=\left(\begin{array}{ll}
a & b \\
c & d
\end{array}\right) \in \Gamma_{0}(N)
$$

We denote by $S_{k}(N, \psi)$ the subspace of $G_{k}(N, \psi)$ consisting of cusp forms and by $S_{x}^{0}(N, \psi)$ the space of new forms in $S_{k}(N, \psi)$. For the trivial character $\psi_{1}$, we put $S_{k}(N)=S_{k}\left(N, \psi_{1}\right)$ and $S_{k}^{0}(N)=S_{k}^{0}\left(N, \psi_{1}\right)$. For a positive integer $n$ prime to $N$, the Hecke operator $T_{n}$ on $S_{x}(N, \psi)$ is defined in the usual way by

$$
f\left|T_{n}=n^{\kappa / 2-1} \sum_{\substack{a d=n \\
b \bmod d}} \psi(a) f\right|\left[\left(\begin{array}{ll}
a & b \\
0 & d
\end{array}\right)\right]_{\kappa} .
$$

For a Dirichlet character $\chi$, we denote by $f_{x}$ the conductor of $\chi$. Let $\chi$ be a primitive character with $\mathfrak{f}_{x}=c$. Then for $f \in S_{\kappa}(N, \psi)$, the twisting operator $R_{x}$ is defined as follows;

$$
f\left|R_{\chi}=\frac{1}{g(\bar{\chi})} \sum_{i \bmod c} \bar{\chi}(i) f\right|\left[\left(\begin{array}{cc}
1 & i / c \\
0 & 1
\end{array}\right)\right]_{\kappa},
$$

where $\mathfrak{g}(\bar{\chi})$ is the Gauss sum for $\bar{\chi}$. Then it is known (c.f. [13]) that $f \mid R_{x}$ belongs to $S_{k}\left(N^{\prime}, \psi \chi^{2}\right)$, where $N^{\prime}$ is the least common multiple of $N, \mathrm{f}_{\psi} \mathrm{f}_{x}$ and $\mathfrak{f}_{x}^{2}$. For a positive divisor $M$ of $N$ such that $(M, N / M)=1$, we choose and fix an element $\gamma_{M}$ of $S L_{2}(Z)$ which satisfies

$$
\gamma_{M} \equiv \begin{cases}\left(\begin{array}{rr}
0 & -1 \\
1 & 0
\end{array}\right) & \left(\bmod M^{4}\right) \\
\left(\begin{array}{rr}
1 & 0 \\
0 & 1
\end{array}\right) & \left(\bmod (N / M)^{4}\right)\end{cases}
$$

and put

$$
\eta_{M}=\gamma_{M}\left(\begin{array}{cc}
M & 0 \\
0 & 1
\end{array}\right)
$$

For $M=N$ and $M=1$, we take respectively

$$
\eta_{N}=\left(\begin{array}{rr}
0 & -1 \\
N & 0
\end{array}\right), \quad \eta_{1}=\left(\begin{array}{ll}
1 & 0 \\
0 & 1
\end{array}\right)
$$


For a positive divisor $M$ of $N$, we denote by $\tilde{M}$ the divisor of $N$ such that the sets of primes which divide $M$ and $\tilde{M}$ are the same and $(\tilde{M}, N / \tilde{M})$ $=1$. For a positive divisor $M$ of $N$, we put $\eta_{M}=\eta_{\tilde{M}}$, and define the operator $W_{M}$ by

$$
f\left|W_{M}=f\right|\left[\eta_{M}\right]_{\kappa} .
$$

Let $\chi$ be a character modulo $N$, and $M$ a divisor of $N$ such that $(M, N / M)$ $=1$. Then $\chi$ can be expressed as $\chi=\chi_{M} \chi_{N / M}$, where $\chi_{M}$ (resp. $\chi_{N / M}$ ) is a character modulo $M$ (resp. $N / M$ ). For a positive divisor $M^{\prime}$ of $N$, we put $\chi_{M^{\prime}}=\chi_{\tilde{M}^{\prime}}$. In this notation, it is known that $f \mid W_{M}$ is contained in $S_{\varepsilon}(N$, $\left.\bar{\psi}_{M} \psi_{N / M}\right)$. These operators $T_{n}, R_{x}$, and $W_{M}$ satisfy the following properties.

LEMMA 1.1. Let $\chi$ be a primitive character, and $M$ a positive divisor of $N$ such that $(M, N / M)=1$. Then for $f \in S_{x}(N, \psi)$, one has

(1) If $n$ is a positive integer prime to $N \mathfrak{f}_{x}$, then

$$
\begin{aligned}
& f\left|T_{n} R_{x}=\bar{\chi}(n) f\right| R_{z} T_{n} \\
& f\left|T_{n} W_{M}=\psi_{M}(n) f\right| W_{M} T_{n} .
\end{aligned}
$$

(2) Suppose $\left(M, \mathfrak{f}_{x}\right)=1$. Then

$$
f\left|R_{x} W_{M}=\bar{\chi}(M) f\right| W_{M} R_{x} .
$$

(3) Let $M^{\prime}$ be a positive divisor of $N$ such that $\left(M^{\prime}, N / M^{\prime}\right)=1$ and $\left(M, M^{\prime}\right)=1$. Then

$$
\begin{aligned}
& f\left|W_{M} W_{M^{\prime}}=\bar{\psi}_{M^{\prime}}(M) f\right| W_{M M^{\prime}} \\
& f \mid W_{M} W_{M}=\psi_{M}(-1) \bar{\psi}_{N / M}(M) f .
\end{aligned}
$$

These properties of $T_{n}, R_{x}$, and $W_{M}$ are given in Atkin-Li [1] and can be verified easily by straightforward computations.

Now we give a definition of the operator $U_{x}$, which is essential to our decomposition of $S_{k}(N, \psi)$. Let $\chi$ be a primitive character with the conductor $\mathfrak{f}_{x}=M$. We assume

$$
\mathfrak{f}_{x}^{2} \mid N \text { and } \mathfrak{f}_{x} \mathfrak{f}_{\psi} \mid N \text {. }
$$

For such a character $\chi$, we define the operator $U_{x}$ by

$$
U_{x}=R_{x} W_{M} R_{x} W_{M} .
$$

For the trivial character $\chi_{1}$, we define $U_{x_{1}}=$ the identity map. Then $U_{x}$ induces a map 


$$
U_{x}: S_{\kappa}(N, \psi) \longrightarrow S_{\kappa}(N, \psi) .
$$

Furthermore, $U_{\chi}$ satisfies the following properties.

Proposition 1.2. The notation being as above, let $f \in S_{x}(N, \psi)$.

(1) If $n$ is a positive integer prime to $N$, then

$$
f\left|T_{n} U_{\chi}=f\right| U_{\chi} T_{n}
$$

(2) Let $\chi^{\prime}$ be a primitive character which satisfies the condition (1.1). Suppose $\left(\mathfrak{f}_{\chi}, \mathfrak{f}_{\chi^{\prime}}\right)=1$. Then

$$
f\left|U_{\chi} U_{\chi^{\prime}}=\bar{\psi}_{M} \bar{\chi}\left(M^{\prime}\right) \bar{\psi}_{M^{\prime}} \bar{\chi}^{\prime}(M) f\right| U_{\chi \chi^{\prime}},
$$

where $M=\mathfrak{f}_{x}$ and $M^{\prime}=\mathfrak{f}_{x^{\prime}}$.

(3) If $\psi$ is the trivial character, then for a positive divisor $L$ of $N$ prime to $\mathfrak{f}_{x}$, it holds

$$
f\left|U_{\chi} W_{L}=f\right| W_{L} U_{x} .
$$

Proof. Let $M=\mathfrak{f}_{x}$, then by (1) of Lemma 1.1, we see

$$
\begin{aligned}
f \mid T_{n} U_{\chi} & =f \mid T_{n} R_{\chi} W_{M} R_{\chi} W_{M} \\
& =\bar{\chi}(n) f \mid R_{\chi} T_{n} W_{M} R_{\chi} W_{M} \\
& =\chi(n) \psi_{M}(n) f \mid R_{\chi} W_{M} T_{n} R_{\chi} W_{M} \\
& =f \mid R_{\chi} W_{M} R_{\chi} W_{M} T_{n} .
\end{aligned}
$$

The assertions (3) and (3) can be proved in a similar way by using Lemma 1.1 , and we omit the proof.

For $M=\mathfrak{f}_{x}$, let $\tilde{M}$ be as above, and put

$$
\tilde{U}_{\chi}=\psi_{\tilde{M}}(-1) \psi_{N / \tilde{M}}(\tilde{M}) \chi(N / \tilde{M}) U_{\chi} .
$$

Then the assertion (2) of the above proposition is equivalent to the following.

Corollary 1.3. If $\mathfrak{f}_{x}$ is prime to $\mathfrak{f}_{x^{\prime}}$, then

$$
\tilde{U}_{\chi} \tilde{U}_{\chi^{\prime}}=\tilde{U}_{\chi \chi^{\prime}}
$$

Proposition 1.4. The notation being as above, then the following assertions hold.

(1) If $f$ is a primitive form in $S_{k}^{0}(N, \psi)$, then $f$ is an eigen-function for $U_{x}$. In particular, $U_{x}$ induces a map

$$
U_{x}: S_{\kappa}^{0}(N, \psi) \longrightarrow S_{\kappa}^{0}(N, \psi) .
$$


(2) Suppose $v_{p}\left(\mathfrak{f}_{\chi} \mathfrak{f}_{\psi}\right)<v_{p}(N)$ and $v_{p}\left(\mathfrak{f}_{\chi}^{2}\right)<v_{p}(N)$ for a prime divisor $p$ of $\mathfrak{f}_{x}$. If $g$ belong to $S_{x}(N / p, \psi)$, then

$$
g \mid U_{x}=0
$$

(3) Let $f$ be a primitive form in $S_{x}^{0}(N, \psi)$. If $f \mid U_{x}=0$ for a character $\chi$ with $\mathfrak{f}_{\chi}=p^{\mu}$, where $p$ is a prime divisor of $N$, then it holds $v_{p}\left(\mathfrak{f}_{\chi} \mathfrak{F}_{\psi}\right)=v_{p}(N)$ or $v_{p}\left(\mathfrak{f}_{\chi}^{2}\right)=v_{p}(N)$, and there exists $g \in S_{x}\left(N / p, \psi \chi^{2}\right)$ such that $f=g \mid R_{\bar{\chi}}$.

(4) If $\psi$ is the trivial character $\psi_{1}$ and $f \in S_{k}^{0}\left(N, \psi_{1}\right)$, then for any divisor $L$ of $N$, it holds

$$
f\left|U_{\chi} W_{L}=f\right| W_{L} U_{\chi} .
$$

Proof. The assertions (1) and (4) easily follows from Prop. 1.2. We shall prove (2) and (3). To prove (2), we may assume $g$ is a primitive form. From the assumption, it follows $g \mid R_{\chi} \in S_{x}\left(N / p, \psi \chi^{2}\right)$. Put $\eta_{M}^{\prime}=\gamma_{M}\left(\begin{array}{cc}M / p & 0 \\ 0 & 1\end{array}\right)$, then $g \mid R_{\chi}\left[\eta_{M}^{\prime}\right]_{\kappa}$ belongs to $S_{x}\left(N / p, \bar{\psi}_{M} \psi_{N / M} \chi^{2}\right)$. Hence $g \mid R_{\chi} W_{M}=g^{\prime}(p z)$ for $g^{\prime} \in$ $S_{k}\left(N / p, \bar{\psi}_{M} \psi_{N / M} \chi^{2}\right)$, and $g \mid R_{\chi} W_{M} R_{\chi}=0$. This proves the assertion (2). Now we prove (3). By the assumption on $\chi$, we have $v_{p}(N) \geq 2$ and $v_{p}\left(\mathfrak{F}_{\psi}\right)<$ $v_{p}(N)$. Hence the $p$-th Fourier coefficient $a_{p}$ of $f$ vanishes, and $f \mid R_{\chi} R_{\bar{\chi}}=$ f. If $f \mid R_{x}$ is a primitive form in $S_{x}^{0}\left(N, \psi \chi^{2}\right)$, then $f \mid R_{\chi} W_{M}$ is also a non-zero constant multiple of a primitive form, and $f \mid R_{\chi} W_{M} R_{x} W_{M} \neq 0$. Hence if $f \mid U_{x}$ $=0$, then $f \mid U_{\chi}$ is not a primitive form in $S_{k}^{0}\left(N, \psi \chi^{2}\right)$, and there exist $g, h$ $\in S_{x}\left(N / p, \psi \chi^{2}\right)$ such that $\left(f \mid R_{x}\right)(z)=g(z)+h(p z)$. Then we have $f=f \mid R_{\chi} R_{\bar{\chi}}$ $=g \mid R_{\bar{\chi}}$. Now we show that $f \mid R_{\chi}$ is a primitive form in $S_{\kappa}^{0}\left(N, \psi \chi^{2}\right)$ if $v_{p}\left(\mathfrak{f}_{\psi} \mathfrak{f}_{\chi}\right)$ $<v_{p}(N)$ and $v_{p}\left(\mathfrak{f}_{x}^{2}\right)<v_{p}(N)$. Otherwise $f \mid R_{x}$ can be written as $f \mid R_{x}=g^{\prime}(z)$ $+h^{\prime}(p z)$ with $g^{\prime}, h^{\prime} \in S_{\star}\left(N / p, \psi \chi^{2}\right)$. Then $f=f \mid R_{\chi} R_{\bar{\chi}} \in S_{x}(N / p, \psi)$, because $v_{p}(N / p) \geq v_{p}\left(\mathfrak{f}_{\psi} \mathfrak{f}_{\chi}\right)$ and $v_{p}(N / p) \geq v_{p}\left(\mathfrak{f}_{\chi}^{2}\right)$. This contradicts to our assumption that $f \in S_{\kappa}^{0}(N, \psi)$.

\section{§2. Formula for $\operatorname{tr} U_{\chi} T_{n}$ and $\operatorname{tr} U_{\chi} W_{L} T_{n}$}

Let $N$ and $\psi$ be as in $\S 1$. For a primitive character $\chi$ which satisfies the condition (1.1), we defined an operator $U_{x}: S_{x}(N, \psi) \longrightarrow S_{x}(N, \psi)$ in $\S 1$. We shall give a formula for $\operatorname{tr} U_{x} T_{n} \mid S_{x}(N, \psi)$. For $M=\mathfrak{f}_{x}$, we write $N=$ $N_{1} N_{2}$, where $N_{1}=\tilde{M}$ and $N_{2}=N / \tilde{M}$. We put

$$
R(N)=\left(\begin{array}{ll}
Z & Z \\
N Z & Z
\end{array}\right)
$$

and for each prime $p$ 


$$
U_{p}=\left(R(N) \otimes Z_{p}\right)^{\times}
$$

For the archimedean prime $\infty$, we put $U_{\infty}=G L_{2}(R)^{+}$. We denote by $U$ the subgroup $\prod_{v} U_{v}$ of $G L_{2}\left(\boldsymbol{Q}_{A}\right)$, where $v$ runs through all places of $\boldsymbol{Q}$. Let $p$ be a prime divisor of $N$ and $\gamma=\left(\begin{array}{ll}a & b \\ c & d\end{array}\right) \in U_{p}$. We define

$$
\tilde{\psi}_{p}(\gamma)=\psi_{p}(d) \text {, }
$$

and for $\gamma \in \prod_{p \mid N} U_{p} \times \prod_{p \nmid N} G L_{2}\left(\boldsymbol{Q}_{p}\right) \times U_{\infty}$

$$
\tilde{\psi}(\gamma)=\prod_{p \mid N} \tilde{\psi}_{p}\left(\gamma_{p}\right)
$$

where $\gamma_{p}$ is the $p$-th component of $\gamma$. For a prime which divides $N_{1}$, we define a subset $\Xi_{p}\left(U_{x}\right)$ of $M_{2}\left(Z_{p}\right)$ by

$$
\Xi_{p}\left(U_{x}\right)=\left\{g \in\left(\begin{array}{ll}
p^{\nu+2 \mu} Z_{p} & p^{\nu+\mu} Z_{p}^{\times} \\
p^{2 \nu+\mu} Z_{p}^{\times} & p^{\nu+2 \mu} Z_{p}^{\times}
\end{array}\right) \mid v_{p}(\operatorname{det} g)=2 \nu+4 \mu\right\}
$$

where $\nu=v_{p}(N)$ and $\mu=v_{p}\left(\mathfrak{f}_{x}\right)$. For $g=\left(\begin{array}{ll}a & b \\ c & d\end{array}\right) \in \Xi_{p}\left(U_{x}\right)$, we put

$$
\tilde{\chi}_{p}(g)=\bar{\chi}_{p}\left(-b c / p^{3 \nu+2 \mu}\right) \bar{\psi}_{p}\left(-d / p^{\nu+2 \mu}\right) .
$$

Then for $\gamma, \gamma^{\prime} \in U_{p}$ and $g \in E_{p}\left(U_{x}\right)$, we see

$$
\tilde{\chi}_{p}\left(\gamma g \gamma^{\prime}\right)=\tilde{\psi}_{p}\left(\gamma \gamma^{\prime}\right)^{-1} \chi_{p}\left(\operatorname{det}\left(\gamma \gamma^{\prime}\right)\right) \tilde{\chi}_{p}(g),
$$

and in particular for $\gamma^{\prime}=\gamma^{-1}$,

$$
\tilde{\chi}_{p}\left(\gamma g \gamma^{-1}\right)=\tilde{\chi}_{p}(g) \text {. }
$$

For $g \in \prod_{p \mid N_{1}} \Xi_{p}\left(U_{x}\right) \times \prod_{p \mid N_{2}} U_{p} \times \prod_{p \nmid N} G L_{2}\left(\boldsymbol{Q}_{p}\right) \times U_{\infty}$, put

$$
\tilde{\chi}(g)=\prod_{p \mid N_{1}} \tilde{\chi}_{p}\left(g_{p}\right) \prod_{p \mid N_{2}} \tilde{\psi}_{p}\left(g_{p}\right)^{-1},
$$

where $g_{p}$ denotes the $p$-th component of $g$. Then by (2.2), we see for $\gamma$, $\gamma^{\prime} \in \prod_{p \mid N} U_{p} \times \prod_{p \nmid N} G L_{2}(\boldsymbol{Q}) \times U_{\infty}$,

$$
\tilde{\chi}\left(\gamma g \gamma^{\prime}\right)=\tilde{\psi}\left(\gamma \gamma^{\prime}\right)^{-1} \prod_{p \mid N_{1}} \chi_{p}\left(\operatorname{det}\left(\gamma_{p} \gamma_{p}^{\prime}\right)\right) \tilde{\chi}(g),
$$

and in particular, if $\gamma, \gamma^{\prime} \in \Gamma_{0}(N)$, then

$$
\tilde{\chi}\left(\gamma g \gamma^{\prime}\right)=\tilde{\psi}\left(\gamma \gamma^{\prime}\right)^{-1} \tilde{\chi}(g)
$$

For rational integers $i, j$, put

$$
\alpha_{i j}=\left(\begin{array}{cc}
M & i \\
0 & M
\end{array}\right) \eta_{M}\left(\begin{array}{cc}
M & j \\
0 & M
\end{array}\right) \eta_{M}
$$


where $M=\mathfrak{f}_{x}$. For a positive integer $n$ prime to $N$, let $\Xi\left(T_{n}\right)=\prod_{p} \Xi_{p}\left(T_{n}\right)$ $\times U_{\infty}$, where

$$
\Xi_{p}\left(T_{n}\right)=\left\{g \in R(N) \otimes Z_{p} \mid v_{p}(\operatorname{det} g)=v_{p}(n)\right\},
$$

and let $\Xi\left(T_{n}\right) \cap G L_{2}(Q)=\bigcup_{k=1}^{d} \Gamma_{0}(N) \beta_{k}$ be a disjoint union.

Lemma 2.1. The notation being as above, let $p$ be a prime divisor of $\mathrm{f}_{x} \quad$ and $\nu=v_{p}(N), \quad \mu=v_{p}\left(\mathfrak{f}_{x}\right)$. Then for $g=\left(\begin{array}{ll}p^{\nu+2 \mu} a & p^{\nu+\mu} b \\ p^{2 \nu+\mu} c & p^{\nu+2 \mu} d\end{array}\right)$ and $g^{\prime}$ $=\left(\begin{array}{ll}p^{\nu+2 \mu} a^{\prime} & p^{\nu+\mu} b^{\prime} \\ p^{2 \nu+\mu} c^{\prime} & p^{\nu+2 \mu} d^{\prime}\end{array}\right)$ in $\Xi_{p}\left(U_{x}\right), U_{p} g=U_{p} g^{\prime}$ if and only if $a / b \equiv a^{\prime} / b^{\prime}$ modulo $p^{\mu}$ and $c / d \equiv c^{\prime} / d^{\prime}$ modulo $p^{\mu}$. If this is the case, $\tilde{\psi}_{p}\left(g g^{\prime-1}\right)=\psi_{p}\left(a^{\prime} d-p^{\nu-2 \mu} b^{\prime} c\right)$.

This can be verified easily by a direct calculation, and we omit the proof.

Lemma 2.2. The notation being as above, let $\Xi\left(U_{x} T_{n}\right)=\prod_{p \mid N_{1}} g_{p}\left(U_{x}\right)$ $\times \prod_{p \nmid N_{1}} \Xi_{p}\left(T_{n}\right) \times U_{\infty}$. Then the union

$$
E\left(U_{x} T_{n}\right) \cap G L_{2}(Q)=\bigcup_{i j} \bigcup_{k=1}^{d} \Gamma_{0}(N) \alpha_{i j} \beta_{k}
$$

is disjoint, where $i$ and $j$ runs through a complete system of representatives of $\left(Z / \mathfrak{f}_{\chi} Z\right)^{\times}$.

Proof. Since $U \cap G L_{2}(\boldsymbol{Q})=\Gamma_{0}(N)$ and $\alpha_{i j} \beta_{k} \in G L_{2}(\boldsymbol{Q})$, it is enough to prove the union $E\left(U_{x} T_{n}\right)=\bigcup_{i j} \bigcup_{k} U \alpha_{i j} \beta_{k}$ is disjoint. We note the union $\prod_{p \nmid N_{1}} \Xi_{p}\left(T_{n}\right)=\bigcup_{k} \prod_{p \nmid N_{1}} U_{p} \beta_{k}$ is disjoint and $\alpha_{i j} \in \prod_{p \nmid N_{1}} U_{p}, \beta_{k} \in \prod_{p \mid N_{1}} U_{p}$. Hence the proof can be reduced to showing the union $\prod_{p \mid N_{1}} \Xi_{p}\left(U_{x}\right)$ $=\bigcup_{i j} \prod_{p \mid N_{1}} U_{p} \alpha_{i j}$ is disjoint. Let $M=\mathfrak{f}_{x}$ and $\tilde{M}=N_{1}$, then

$$
\alpha_{i j} \equiv\left\{\begin{array}{ll}
\left(\begin{array}{cc}
i j \tilde{M}^{2}-\tilde{M} M^{2} & -i \tilde{M} M \\
j \tilde{M}^{2} M & -\tilde{M} M^{2}
\end{array}\right) & \left(\bmod \tilde{M}^{4}\right) \\
\left(\tilde{M}^{2} M^{2}\right. & j \tilde{M} M+i M \\
0 & M^{2}
\end{array},\right.
$$

and by the definition of $\Xi_{p}\left(U_{\chi}\right), \alpha_{i j} \in \prod_{p \mid N_{1}} \Xi_{p}\left(U_{x}\right)$. By Lemma 2.1, for integers $i, j, i^{\prime}, j^{\prime}$ prime to $N_{1}$, we see

$$
U_{p} \alpha_{i j}=U_{p} \alpha_{i^{\prime} j^{\prime}} \Longleftrightarrow i \equiv i^{\prime}, j \equiv j^{\prime} \quad\left(\bmod p^{\mu}\right) .
$$

Hence the right side of the union is disjoint. We show $\prod_{p \mid N_{1}} \Xi_{p}\left(U_{x}\right)$ $\subset \cup_{i j} \prod_{p \mid N_{1}} U_{p} \alpha_{i j}$. For a prime $p$ which divides $N_{1}$, let $g=\left(\begin{array}{cc}p^{\nu+2 \mu} a & p^{\nu+\mu} b \\ p^{2 \nu+\mu} c & p^{\nu+2 \mu} d\end{array}\right)$ 
$\in \Xi_{p}\left(U_{x}\right)$. If we put $\tilde{M}=p^{\nu} \tilde{M}^{\prime}, M=p^{u} M^{\prime}$ and take two integers $i, j$ which satisfy

$$
\left\{\begin{array}{rl}
\left(i j \tilde{M}^{\prime 2}-M^{\prime} M^{\prime 2}\right) b & \equiv-i \tilde{M}^{\prime} M^{\prime} a \\
j \tilde{M}^{\prime 2} M^{\prime} d & \equiv-\tilde{M}^{\prime} M^{2} c
\end{array} \quad\left(\bmod p^{\mu}\right),\right.
$$

then by Lemma 2.1, we have $U_{p} g=U_{p} \alpha_{i j}$. Such $i$ and $j$ are determined uniquely modulo $p^{\mu}$, because $a d-b c \not \equiv 0(\bmod p)$. Our assertion follows from this.

As a corollary of this Lemma, we obtain

Corollary 2.3. The notation being as above, let $f \in S_{\kappa}(N, \psi)$. Then it holds

$$
\begin{aligned}
& f\left|U_{\chi} T_{n}=C \sum_{g \in \Gamma_{0}(N) \backslash \varepsilon\left(U_{\chi} T_{n}\right) \cap G L_{2}(Q)} \tilde{\chi}(g) f\right|[g]_{\kappa} \\
& C=\frac{\chi \psi(n)}{g(\bar{\chi})^{2}} \prod_{p \mid N_{1}} \chi_{p}\left(A_{p}\right) \psi_{p}\left(B_{p}\right) \prod_{p \mid N_{2}} \psi_{p}\left(M^{2}\right),
\end{aligned}
$$

where g runs through a complete system of representatives of the left cosets of $\Xi\left(U_{x} T_{n}\right) \cap G L_{2}(Q)$ by $\Gamma_{0}(N)$ and for a prime divisor $p$ of $N_{1}, A_{p}=$ $\tilde{M}^{3} M^{2} / p^{3 \nu+2 \mu}$ and $B_{p}=\tilde{M} M^{2} / p^{\nu+2 \mu}$ with $\nu=v_{p}(N)$ and $\mu=v_{p}\left(\mathfrak{f}_{x}\right)$.

Proof. We note the right hand side is independent of the choice of the representatives because of (2.5). We may assume $\beta_{k}$ is of the form $\left(\begin{array}{ll}a & b \\ 0 & d\end{array}\right)$. Since we have

$$
\alpha_{i j} \beta_{k} \equiv\left\{\begin{array}{cc}
\left(\begin{array}{cc}
a\left(i j \tilde{M}^{2}-\tilde{M} M^{2}\right) & b\left(i j \tilde{M}^{2}-\tilde{M} M^{2}\right)-i d \tilde{M} M \\
a j \tilde{M}^{2} M & b j \tilde{M}^{2} M-d \tilde{M} M^{2}
\end{array}\right) & \left(\bmod \tilde{M}^{4}\right) \\
\left(\begin{array}{cc}
a \tilde{M}^{2} M^{2} & b \tilde{M}^{2} M^{2}+d(j \tilde{M} M+i M) \\
0 & d M^{2}
\end{array}\right) & \left(\bmod (N / \tilde{M})^{4}\right)
\end{array}\right.
$$

we see $\tilde{\chi}\left(\alpha_{i j} \beta_{k}\right)=\bar{\chi}(i j) \psi(a) C^{-1}$. By the definition of $U_{x}$ and $T_{n}$, we obtain our corollary.

By means of Eichler-Selberg's trace formula (c.f. [6], [8], [10], [12]) and a result of Hijikata [8], we can express trace of $U_{x} T_{n}$ on $S_{k}(N, \psi)$ in an explicit way. Let us introduce some notations. For two rational integers $s, n$, put $\Phi(X)=X^{2}-s X+n, K(\Phi)=Q[X] /(\Phi(X))$, and denote by $\tilde{X}$ the class containing $X$. For a prime $p$, let $\nu=v_{p}(N)$ and $K(\Phi)_{p}=K(\Phi) \otimes \boldsymbol{Q}_{p}$. If we define $R_{p}(\nu)=\left(\begin{array}{ll}Z_{p} & Z_{p} \\ p^{\nu} Z_{p} & Z_{p}\end{array}\right)$, then $R(N) \otimes Z_{p}=R_{p}(\nu)$. For $\alpha$ in $G L_{2}\left(Q_{p}\right)$ or $G L_{2}(R)$, we denote by $f_{\alpha}(X)$ the minimal polynomial of $\alpha$. For a $Z_{p^{-}}$ order $\Lambda_{p}$ of $K(\Phi)_{p}$, we define 


$$
C_{p}\left(\nu, \Phi, \Lambda_{p}\right)=\left\{\alpha \in R_{p}(\nu) \mid f_{\alpha}=\Phi, \varphi_{\alpha}\left(\Lambda_{p}\right)=\boldsymbol{Q}_{p}[\alpha] \cap R_{p}(\nu)\right\},
$$

where $\varphi_{\alpha}$ denotes the isomorphism from $K(\Phi)_{p}$ to $Q_{p}[\alpha]$ such that $\varphi_{\alpha}(\bar{X})=$ $\alpha$. For $\Lambda_{p}$ which contains $Z_{p}[\tilde{X}]$, we define also the following sets;

$$
\begin{aligned}
& \left.\Omega_{p}\left(\nu, \Phi, \Lambda_{p}\right)=\left\{\xi \in Z_{p} \mid \Phi(\xi) \equiv 0\left(\bmod P^{\nu+2 \rho}\right)\right)\right\} \\
& \Omega_{p}^{\prime}\left(\nu, \Phi, \Lambda_{p}\right)=\left\{\begin{array}{c}
\left\{\eta \in Z_{p} \mid \Phi(\eta) \equiv 0\left(\bmod p^{\nu+2 \rho+1}\right)\right\}, \\
\text { if } p^{-2 \rho}\left(s^{2}-4 n\right) \equiv 0(\bmod p) \text { and } \nu>0 \\
\phi, \quad \text { otherwise },
\end{array}\right.
\end{aligned}
$$

where $\rho$ is the non-negative integer such that $\left[\Lambda_{p}: Z_{p}[\tilde{X}]\right]=p^{\rho}$. We denote by $\tilde{\Omega}_{p}\left(\nu, \Phi, \Lambda_{p}\right)$ (resp. $\tilde{\Omega}_{p}^{\prime}\left(\nu, \Phi, \Lambda_{p}\right)$ ) a complete system of representatives of $\Omega_{p}\left(\nu, \Phi, \Lambda_{p}\right)$ (resp. $\left.\Omega_{p}^{\prime}\left(\nu, \Phi, \Lambda_{p}\right)\right)$ modulo $p^{\nu+2 \rho}$. For $\xi \in \Omega_{p}\left(\nu, \Phi, \Lambda_{p}\right)$ and $\eta \in$ $\Omega_{p}\left(\nu, \Phi, \Lambda_{p}\right)$ we define elements $\varphi_{\xi}(\tilde{X})$ and $\varphi_{\eta}^{\prime}(\tilde{X})$ in $C_{p}\left(\nu, \Phi, \Lambda_{p}\right)$ by

$$
\begin{aligned}
\varphi_{\xi}(\tilde{X}) & =\left(\begin{array}{cc}
\xi & p^{\rho} \\
-p^{-\rho} \Phi(\xi) & s-\xi
\end{array}\right) \\
\varphi_{\eta}^{\prime}(\tilde{X}) & =\left(\begin{array}{cc}
s-\eta & -p^{-\nu-\rho} \Phi(\eta) \\
p^{\nu+\rho} & \eta
\end{array}\right) .
\end{aligned}
$$

We define a map

$$
\varphi: \Omega_{p}\left(\nu, \Phi, \Lambda_{p}\right) \cup \Omega_{p}^{\prime}\left(\nu, \Phi, \Lambda_{p}\right) \longrightarrow C_{p}\left(\nu, \Phi, \Lambda_{p}\right)
$$

by $\varphi(\xi)=\varphi_{\xi}(\tilde{X})$ for $\xi \in \Omega_{p}\left(\nu, \Phi, \Lambda_{p}\right)$ and $\varphi(\eta)=\varphi_{\eta}^{\prime}(\tilde{X})$ for $\eta \in \Omega_{p}^{\prime}\left(\nu, \Phi, \Lambda_{p}\right)$. In these notations, we have

Lemma 2.4. The notation being as above, let $\Phi(X)=X^{2}-s X+N^{2}{ }_{x}^{4} n$ and for a prime $p$, let $\Lambda_{p}$ a $Z_{p}$-order of $K(\Phi)_{p}$ such that $\Lambda_{p} \supset Z_{p}[\tilde{X}]$. Then the followings hold.

(1) If $p$ does not divide $N$, then $\varphi$ induces a bijective map

$$
\varphi: \Omega_{p}\left(0, \Phi, \Lambda_{p}\right) \longrightarrow C_{p}\left(0, \Phi, \Lambda_{p}\right) \cap \Xi_{p}\left(T_{n}\right) / \widetilde{U}_{p},
$$

and $\left|\widetilde{\Omega}_{p}\left(0, \Phi, \Lambda_{p}\right)\right|=1$.

(2) If $p$ divides $N_{2}$, then $\varphi$ induces a bijective map

$$
\varphi: \Omega_{p}\left(\nu, \Phi, \Lambda_{p}\right) \cup \Omega_{p}^{\prime}\left(\nu, \Phi, \Lambda_{p}\right) \longrightarrow C_{p}\left(\nu, \Phi, \Lambda_{p}\right) \cap U_{p} /_{\widetilde{U_{p}}},
$$

where $\nu=v_{p}(N)$.

(3) If $p$ divides $N_{1}$, then $C_{p}\left(\nu, \Phi, \Lambda_{p}\right) \cap \Xi_{p}\left(U_{x}\right) \neq \phi$ only if $s \equiv 0\left(\bmod p^{\nu+2 \mu}\right)$ and $\rho=\nu+\mu$, and for $\Phi$ with $s \equiv 0\left(\bmod p^{\nu+2 \mu}\right)$ and $\Lambda_{p}$ with $\rho=\nu+\mu, \varphi$ induces a bijective map 


$$
\varphi:\left.\widetilde{\Omega}_{p} \longrightarrow C_{p}\left(\nu, \Phi, \Lambda_{p}\right) \cap \Xi_{p}\left(U_{z}\right)\right|_{\widetilde{U}_{p}},
$$

where $\nu=v_{p}(N), \mu=v_{p}\left(\mathfrak{f}_{x}\right)$ and

$$
\tilde{\Omega}_{p}=\left\{\begin{array}{c}
\left\{\xi \in \tilde{\Omega}_{p}\left(\nu, \Phi, \Lambda_{p}\right) \mid \Phi(\xi) \neq 0\left(\bmod p^{3 \nu+2 \mu+1}\right)\right\} \text { if } \nu \neq 2 \mu \\
\left\{\xi \in \tilde{\Omega}_{p}\left(\nu, \Phi, \Lambda_{p}\right) \mid \Phi(\xi) \neq 0\left(\bmod p^{3 \nu+2 \mu+1}\right),\right. \\
\left.s \neq \xi\left(\bmod p^{\nu+2 \mu+1}\right)\right\} \quad \text { if } \nu=2 \mu .
\end{array}\right.
$$

Proof. The assertions (1) and (2) are contained in Hijikata [8]. We prove (3). The theorem of Hijikata quoted in [11] as Th. 2.4 says that for $\Lambda_{p}$ containing $Z_{p}[\tilde{X}], \varphi$ gives a bijective map

$$
\varphi: \Omega_{p}\left(\nu, \Phi, \Lambda_{p}\right) \cap \Omega_{p}^{\prime}\left(\nu, \Phi, \Lambda_{p}\right) \longrightarrow C_{p}\left(\nu, \Phi, \Lambda_{p}\right) / \tilde{U}_{p}
$$

By the definition of $\Xi_{p}\left(U_{x}\right)$, we see $s \equiv 0\left(\bmod p^{\nu+2 \mu}\right)$ if $C_{p}\left(\nu, \Phi, \Lambda_{p}\right) \cap \Xi_{p}\left(U_{x}\right)$ is not empty. If $\varphi_{\eta}^{\prime}(\tilde{X}) \in \Xi_{p}\left(U_{x}\right)$ for $\eta \in \Omega_{p}^{\prime}\left(\nu, \Phi, \Lambda_{p}\right)$, it must hold $\nu+\rho=$ $2 \nu+\mu$ and $\nu+\mu=v_{p}(\Phi(\eta))-\nu-\rho$, hence $\rho=\nu+\mu$ and $v_{p}(\Phi(\eta))=3 \nu+2 \mu$. But if $\rho=\nu+\mu$, then $\eta$ saitsfies $\Phi(\eta) \equiv 0\left(\bmod p^{3 \nu+2 \mu+1}\right)$ hence $\varphi_{\eta}^{\prime}(X) \notin \Xi_{p}\left(U_{x}\right)$. Assume $\varphi_{\xi}(\tilde{X}) \in \Xi_{p}\left(U_{x}\right)$ for $\xi \in \Omega_{p}\left(\nu, \Phi, \Lambda_{p}\right)$. Then as above, we have $\rho=\nu+\mu$ and $v_{p}(\Phi(\xi))=3 \nu+2 \mu$. When these conditions are satisfied, $\varphi_{\xi}(\tilde{X}) \in \Xi_{p}\left(U_{z}\right)$ if and only if $\xi \neq \equiv s\left(\bmod p^{\nu+2 \mu+1}\right)$. We note the last condition is always satisfied if $\nu \neq 2 \mu$. For otherwise, put $s=p^{\nu+2 \mu} s^{\prime}$ and $\xi=p^{\nu+2 \mu}\left(s^{\prime}+p \xi^{\prime}\right)$, then we have

$$
p^{2 \nu+4 \mu}\left(s^{\prime} p \xi^{\prime}+p^{2} \xi^{\prime 2}+n\right) \equiv 0\left(\bmod p^{3 \nu+2 \mu}\right) .
$$

Since $n$ is prime to $p$, this condition is satisfied only if $\nu=2 \mu$. This proves the assertion (3).

By means of this Lemma, in the same way as in $\S 2$ of [11], we obtain the following formula for $\operatorname{tr} U_{x} T_{n}$.

THEOREM 2.5. The notation being as above, let $n$ be a positive integer prime to $N, \kappa \geq 2$, and $C$ the constant in Cor. 2.2. Then it holds

$$
\operatorname{tr} U_{x} T_{n} \mid S_{x}(N, \psi)=C\left(t_{e}+t_{h}+t_{p}\right)
$$

where $t_{e}, t_{h}$ and $t_{p}$ are given as follows.

(1) $t_{e}=-\frac{1}{2} \sum_{s} \frac{\alpha^{\kappa-1}-\beta^{\kappa-1}}{\alpha-\beta} \sum_{f} \prod_{p \mid N} c_{p}(s, f) h\left(\mathfrak{f}_{\chi}^{2}\left(s^{2}-4 n\right) / f^{2}\right)$.

Here $s$ runs through all integers such that $s^{2}-4 n<0$, and $f$ runs through all positive integers which satisfy the condition $f^{2} \mid\left(s^{2}-4 n\right),\left(f, \mathfrak{f}_{x}\right)=1$, and $\mathfrak{f}_{x}^{2}\left(s^{2}-4 n\right) / f^{2} \equiv 0$ or $1(\bmod 4)$. For a negative integer $D$ such that $D \equiv 0$ 
or $1(\bmod 4), h(D)$ denotes the class number of the order of $Q(\sqrt{D})$ with the discriminant $D$. $\alpha$ and $\beta$ are the two roots of $F_{s}(X)=X^{2}-s X+n=0$. The number $c_{p}(s, f)$ is given by

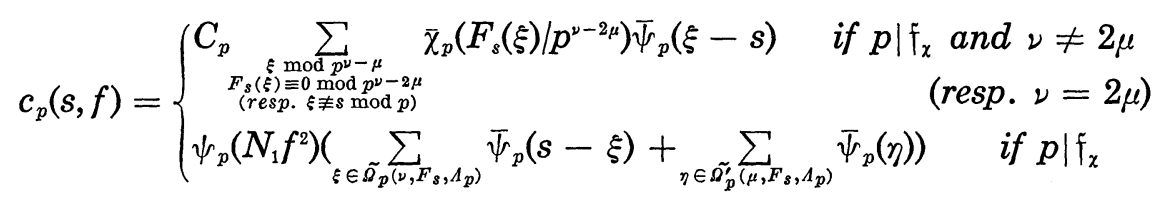

where $\Lambda_{p}$ is the order of $K\left(F_{s}\right)$ such that $\left[\Lambda_{p}: Z_{p}[\tilde{X}]\right]=p^{\rho}$ for $\rho=v_{p}(f)$, and $C_{p}=\bar{\chi}_{p}\left(N_{1}^{2} \mathfrak{\digamma}_{\chi}^{4} / p^{2 \nu+4 \mu}\right) \bar{\psi}_{p}\left(N_{1} \mathfrak{f}_{\chi}^{2} / p^{\nu+2 \mu}\right)$ for $\nu=v_{p}(N)$ and $\mu=v_{p}\left(\mathfrak{F}_{\chi}\right)$.

(2) $\quad t_{h}=-\sum_{d} \frac{d^{k-1}}{n / d-d} \sum_{f} \prod_{p \mid N} c_{p}(d+n / d, f) \varphi\left(\tilde{f}_{x}(n / d-d) / f\right)$.

Here $d$ runs through all positive integers such that $0<d<\sqrt{n}, d \mid n$, and $f$ runs through all positive integers satisfying $f \mid(n / d-d)$ and $\left(f, \mathfrak{f}_{x}\right)=1$. $c_{p}(d+n / d, f)$ is given by (2.6) for $s=d+n / d$, and $\varphi$ is the Euler function.

(3) If there exists a prime divisor $p$ of $\mathrm{f}_{x}$ such that $v_{p}(N)$ is odd, then $t_{p}=0$. Otherwise we have

$$
t_{p}=-\frac{n^{(x-1) / 2}}{2} \frac{\mathfrak{f}_{x}}{N} \delta(n) \sum_{\substack{m \\\left(m, f_{x}\right)=1}} \prod_{p \mid N} c_{p}(m),
$$

where $c_{p}(m)=c_{p}(2 \sqrt{n}, m)$ for $p$ which divides $N$, and $\delta(n)=1$ or 0 according as $n$ is a square or not.

In the rest of this section, we assume $\psi$ is the trivial character. Then for a divisor $L$ of $N$ such that $(L, N / L)=1, U_{x} W_{L}$ acts on $S_{x}(N)$, and we can give a formula for $\operatorname{tr} U_{z} W_{L} T_{n}$. We write $N=M_{1} M_{2} M_{3} M_{4}$ in such a way $N_{1}=M_{1} M_{2}$ and $L=M_{2} M_{3}$. For a prime $p$ which divides $M_{2}$, we define a subset $E_{p}\left(U_{x} W_{L}\right)$ of $R(N) \otimes Z_{p}$ by

$$
\Xi_{p}\left(U_{\chi} W_{L}\right)=\left\{g \in\left(\begin{array}{ll}
p^{2 \nu+\mu} Z_{p}^{\times} & p^{\nu+2 \mu} Z_{p} \\
p^{2 \nu+2 \mu} Z_{p}^{\times} & p^{2 \nu+\mu} Z_{p}^{\times}
\end{array}\right) \mid v_{p}(\operatorname{det} g)=3 \nu+4 \mu\right\},
$$

and for a prime divisor $p$ of $M_{3}$, put

$$
\Xi_{p}\left(W_{L}\right)=\left(\begin{array}{ll}
p^{\nu} Z_{p} & Z_{p}^{\times} \\
p^{\nu} Z_{p}^{\times} & p^{\nu} Z_{p}
\end{array}\right)
$$

For $g=\left(\begin{array}{ll}a & b \\ c & d\end{array}\right) \in \Xi_{p}\left(U_{z} W_{L}\right)$, we put

$$
\tilde{\chi}_{p}^{\prime}(g)=\bar{\chi}_{p}\left(a d / p^{4 \nu+2 \mu}\right)
$$


where $\nu=v_{p}(N), \mu=v_{p}\left(\mathfrak{f}_{x}\right)$. Then for $\gamma, \gamma^{\prime} \in U_{p}$, we see

$$
\chi_{p}^{\prime}\left(\gamma g \gamma^{\prime}\right)=\chi_{p}\left(\operatorname{det}\left(\gamma \gamma^{\prime}\right)\right) \chi_{p}^{\prime}(g) \text {. }
$$

We define a union of $U$-double cosets $\Xi\left(U_{x} W_{L} T_{n}\right)$ by

$$
\Xi\left(U_{\chi} W_{L} T_{n}\right)=\prod_{p \mid M_{1}} \Xi_{p}\left(U_{\chi}\right) \prod_{p \mid M_{2}} \Xi_{p}\left(U_{\chi} W_{L}\right) \prod_{p \mid M_{3}} \Xi_{p}\left(W_{L}\right) \prod_{p \nmid M_{1} M_{2} M_{3}} \Xi_{p}\left(T_{n}\right) \times U_{\infty},
$$

and for $g \in \Xi\left(U_{x} W_{L} T_{n}\right)$, put

$$
\tilde{\chi}^{\prime}(g)=\prod_{p \mid M_{1}} \tilde{\chi}_{p}\left(g_{p}\right) \prod_{p \mid M_{2}} \tilde{\chi}_{p}^{\prime}\left(g_{p}\right),
$$

where $g_{p}$ is the $p$-th component of $g$. Corresponding to Lemma 2.2, we have

Lemma 2.6. The notation being as in Lemma 2.2, for a divisor $L$ of $N$ with $(L, N / L)=1$, the union

$$
\Xi\left(U_{\chi} W_{L} T_{n}\right) \cap G L_{2}(Q)=\bigcup_{i j} \bigcup_{k=1}^{d} \Gamma_{0}(N) \alpha_{i j} \eta_{L} \beta_{k}
$$

is disjoint, where $i$ and $j$ runs through a complete system of representatives of $\left(Z / \mathfrak{f}_{x} Z\right)^{\times}$.

Proof. As in the proof of Lemma 2.2, it is enough to prove the union $\prod_{p \mid M_{1}} \Xi_{p}\left(U_{z}\right) \prod_{p \mid M_{2}} \Xi_{p}\left(U_{z} W_{L}\right) \prod_{p \mid M_{3}} \Xi_{p}\left(W_{L}\right)=\bigcup_{i j} \prod_{p \mid M_{1} M_{2} M_{3}} U_{p} \alpha_{i j} \eta_{L}$ is disjoint. But this follows easily from the proof of Lemma 2.2 and the fact that $\Xi_{p}\left(U_{x} W_{L}\right)=\Xi_{p}\left(U_{x}\right) \eta_{L}$ and $\Xi_{p}\left(W_{L}\right)=U_{p} \eta_{L}$

Corollary 2.7. The notation being as above, then for $f \in S_{\kappa}(N)$, it holds

$$
\begin{aligned}
& f\left|U_{\chi} W_{L} T_{n}=C^{\prime} \sum_{g \in \Gamma_{0}(N) \backslash \varepsilon\left(U_{\chi} W_{L} T_{n}\right) \cap G L_{2}(Q)} \chi^{\prime}(g) f\right|[g]_{k} \\
& C^{\prime}=\chi(n) \prod_{p \mid M_{1}} \chi_{p}\left(A_{p}^{\prime}\right) \prod_{p \backslash M_{2}} \chi_{p}\left(B_{p}^{\prime}\right) / g(\bar{\chi})^{2}
\end{aligned}
$$

where $A_{p}^{\prime}=L N_{1}^{3} \mathfrak{f}_{x}^{3} / p^{3 \nu+2 \mu}$ and $B_{p}^{\prime}=L N_{1}^{3} \mathfrak{f}_{\chi}^{2} / p^{4 \nu+2 \mu}$ for $\nu=v_{p}(N)$ and $\mu=v_{p}\left(\mathfrak{f}_{\chi}\right)$.

Proof. The right hand side of the above equality is independent of the choice of the representatives because of (2.2) and (2.8). If $\beta_{k}$ is of the form $\left(\begin{array}{ll}a & b \\ 0 & d\end{array}\right)$, then we see

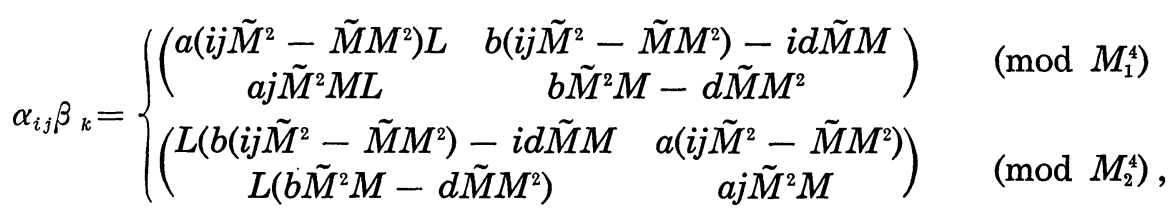


where $\tilde{M}=N_{1}$ and $M=\mathfrak{f}_{x}$. Hence we have

$$
\tilde{\chi}^{\prime}\left(\alpha_{i j} \beta_{k}\right)=\bar{\chi}(i j) C^{\prime-1} .
$$

Our assertion follows from this and Lemma 2.6.

To express $\operatorname{tr} U_{x} W_{L} T_{n}$ in an explicit way, we prove

Lemma 2.8. Let $\Phi(X)=X^{2}-s X+M_{1}^{2} M_{2}^{2} L f_{x}^{4} n$, and for a prime divisor $p$ of $N$, let $\nu=v_{p}(N)$ and $\mu=v_{p}\left(\mathfrak{f}_{\chi}\right)$. Then for an order $\Lambda_{p}$ of $K(\Phi)_{p}$ containint $Z_{p}[\tilde{X}]$, the followings hold.

(1) For $p$ dividing $M_{3}, C_{p}\left(\nu, \Phi, \Lambda_{p}\right) \cap \Xi_{p}\left(W_{L}\right) \neq \phi$ only if $s \equiv 0\left(\bmod p^{\nu}\right)$ and $\Lambda_{p}=Z_{p}[\tilde{X}]$. When this condition is satisfied, one has

$$
\left|C_{p}\left(\nu, \Phi, \Lambda_{p}\right) \cap \Xi_{p}\left(W_{L}\right)\right|_{\widetilde{U}_{p}} \mid=1
$$

(2) For $p$ dividing $M_{2}, C_{p}\left(\nu, \Phi, \Lambda_{p}\right) \cap \Xi_{p}\left(U_{x} W_{L}\right) \neq \phi$ only if $s \equiv 0$ $\left(\bmod p^{2 \nu+\mu}\right)$ and $\left[\Lambda_{p}: Z_{p}[\tilde{X}]\right]=p^{\rho}$, where $\rho=\nu+2 \mu$. When this condition is satisfied, $\varphi$ induces a bijective map

$$
\varphi: \tilde{\Omega}_{p}^{\prime} \longrightarrow C_{p}\left(\nu, \Phi, \Lambda_{p}\right) \cap \Xi_{p}\left(U_{x} W_{L}\right) /_{\widetilde{U}_{p}},
$$

where $\tilde{\Omega}_{p}$ is a complete system of representatives modulo $p^{2 \nu+2 \mu}$ of the set $\left\{p^{2 \nu+\mu} \xi \mid \xi \in Z_{p}^{\times}, \xi \not \equiv s / p^{2 \nu+\mu}(\bmod p)\right\}\left(\subset \Omega_{p}\left(\nu, \Phi, \Lambda_{p}\right)\right)\left(\operatorname{resp} .\left\{p^{2 \nu+\mu} \xi \mid \xi \in Z_{p}^{\times}, \xi \not \equiv\right.\right.$ $\left.s / p^{2 \nu+\mu}(\bmod p), \Phi\left(p^{2 \nu+\mu} \xi\right) \not \equiv 0\left(\bmod p^{3 \nu+4 \mu+1}\right)\right\}\left(\subset \Omega_{p}\left(\nu, \Phi, \Lambda_{p}\right)\right) \cup\left\{p^{2 \nu+\mu} \eta \mid \eta \in Z_{p}^{\times}\right.$, $\left.\left.\eta \not \equiv s / p^{2 \nu+\mu}(\bmod p), \Phi\left(p^{2 \nu+\mu} \eta\right) \equiv 0\left(\bmod p^{3 \nu+4 \mu+1}\right)\right\}\left(\subset \Omega_{p}^{\prime}\left(\nu, \Phi, \Lambda_{p}\right)\right)\right)$ if $\nu>2 \mu(\operatorname{resp}$. if $\nu=2 \mu)$.

Proof. The assertion (1) is contained in Yamauchi [18]. If $C_{p}\left(\nu, \Phi, \Lambda_{p}\right)$ $\cap \Xi_{p}\left(U_{x} W_{L}\right) \neq \phi$, then we see that $s \equiv 0\left(\bmod p^{2 \nu+\mu}\right)$ and $\left[\Lambda_{p}: Z_{p}[\tilde{X}]\right]=p^{\rho}$, where $\rho=\nu+2 \mu$. Assume this condition is satisfied. First we treat the case where $\nu>2 \mu$. In this case, we note $v_{p}(b)=\nu+2 \mu$ for $g=\left(\begin{array}{ll}a & b \\ c & d\end{array}\right) \epsilon$ $\Xi_{p}\left(U_{x} W_{L}\right)$, hence $\varphi_{\eta}^{\prime}(\tilde{X}) \notin \Xi_{p}\left(U_{x} W_{L}\right)$. If $\varphi_{\xi}(\tilde{X}) \in \Xi_{p}\left(U_{x} W_{L}\right)$ for $\xi \in \Omega_{p}\left(\nu, \Phi, \Lambda_{p}\right)$, then $\xi$ is of the form $p^{2 \nu+\mu} \xi^{\prime}$ with $\xi^{\prime} \in Z_{p}$. We note $v_{p}\left(\Phi\left(p^{2 \nu+\mu} \xi^{\prime}\right)\right)=3 \nu+4 \mu$ for $\xi^{\prime} \in Z_{p}$. Hence $\xi=p^{2 \nu+\mu} \xi^{\prime} \in \Omega_{p}\left(\nu, \Phi, \Lambda_{p}\right)$ for $\xi^{\prime} \in Z_{p}$, and $\varphi_{\xi}(\tilde{X}) \in \Xi_{p}\left(U_{x} W_{L}\right)$ if and only if $\xi \not \equiv 0\left(\bmod p^{2 \nu+\mu+1}\right)$ and $s-\xi \not \equiv 0\left(\bmod p^{2 \nu+\mu+1}\right)$. This prove the case $\nu>2 \mu$. Next assume $\nu=2 \mu$. Also in this case, if $\varphi_{\xi}(\tilde{X}) \in \Xi_{p}\left(U_{x} W_{L}\right)$ (resp. $\varphi_{\eta}^{\prime}(\tilde{X}) \in \Xi_{p}\left(U_{x} W_{L}\right)$ ), then $\xi=p^{2 \nu+\mu} \xi^{\prime}$ with $\xi^{\prime} \in Z_{p}$ (resp. $\eta=p^{2 \nu+\mu} \eta^{\prime}$ with $\left.\eta^{\prime} \in Z_{p}\right)$. For $\xi^{\prime} \in Z_{p}$, put $\xi=p^{2 \nu+\mu} \xi^{\prime}$, then $v_{p}(\Phi(\xi)) \geq 3 \nu+4 \mu$. Hence $\xi \in$ $\Omega_{p}\left(\nu, \Phi, \Lambda_{p}\right)$, and $\varphi_{\xi}(\tilde{X}) \in \Xi_{p}\left(U_{x} W_{L}\right)$ if and only if $\xi \neq \equiv\left(\bmod p^{2 \nu+\mu+1}\right), s-\xi$ $\not \equiv 0\left(\bmod p^{2 \nu+\mu+1}\right)$ and $\Phi(\xi) \not \equiv 0\left(\bmod p^{3 \nu+4 \mu+1}\right)$. For $\eta=p^{2 \nu+\mu} \eta^{\prime}$ with $\eta^{\prime} \in Z_{p}$, 
$\eta \in \Omega_{p}^{\prime}\left(\nu, \Phi, \Lambda_{p}\right)$ if and only if $\Phi(\eta) \equiv 0\left(\bmod p^{3 \nu+4 \mu+1}\right)$, and for such $\eta^{\prime} \in Z_{p} \varphi_{\eta}^{\prime}(\tilde{X})$ $\in \Xi_{p}\left(U_{x} W_{L}\right)$ if and only if $\eta \not \equiv 0\left(\bmod p^{2 \nu+\mu+1}\right)$ and $s-\eta \not \equiv 0\left(\bmod p^{2 \nu+\mu+1}\right)$. Our assertion follows from this.

By means of this Lemma, in the similar way as in $\S 3$ of [11], we obtain the following.

TheOREM 2.9. The notation being as above, let $L$ be a divisor of $N$ such that $(L, N / L)=1$. We write $f_{x}=F_{1} F_{2}$, where $F_{1}=\left(\mathfrak{f}_{x}, M_{1}\right)$ and $F_{2}=\left(\mathfrak{f}_{x}, M_{2}\right)$. Then we have

$$
\operatorname{tr} U_{\chi} W_{L} T_{n} \mid S_{\kappa}(N)=C^{\prime}\left(t_{e}+t_{h}+t_{p}\right),
$$

where $C^{\prime}$ is the constant in Cor. 2.7, and $t_{e}, t_{h}$ and $t_{p}$ are given as follows.

(1) $t_{e}=-\frac{1}{2} \sum_{s} \frac{\alpha^{\kappa-1}-\beta^{\kappa-1}}{\alpha-\beta}\left(L F_{2}^{4}\right)^{1-\kappa / 2} \sum_{f} \prod_{p \mid M_{1} M_{2} M_{4}} c_{p}^{\prime}(s, f)$

$$
\times h\left(F_{1}^{2}\left(L^{2} F_{2}^{-2} s^{2}-4 L n\right) / f^{2}\right) .
$$

Here $s$ runs through all integers such that $L^{2} F_{2}^{-2} s^{2}-4 L n<0$, and $f$ runs through all positive integers which satisfy the condition $f^{2} \mid\left(L^{2} F_{2}^{-2} s^{2}-4 L n\right)$, $\left(f, \mathfrak{F}_{x} L\right)=1$ and $F_{1}^{2}\left(L^{2} F_{2}^{-2} s^{2}-4 L n\right) / f^{2} \equiv 0$ or $1(\bmod 4)$. For $s$, put $G_{s}(X)=$ $X^{2}-L F_{2} s X+L F_{2}^{4} n$, then $\alpha$ and $\beta$ are the two roots of $G_{s}(X)=0$. The number $c_{p}^{\prime}(s, f)$ is given by

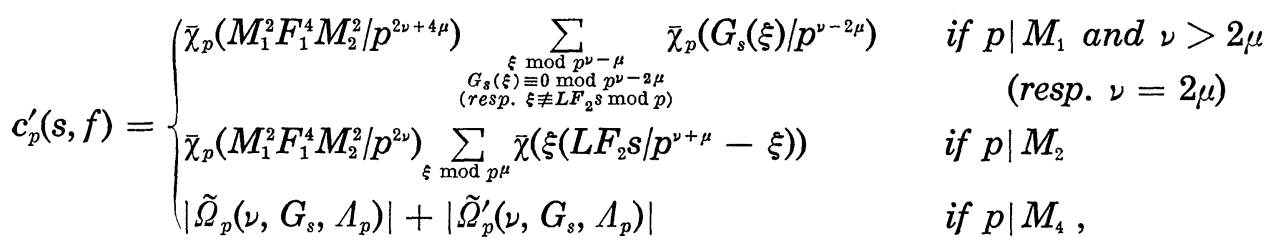

where $\nu=v_{p}(N), \mu=v_{p}\left(\mathfrak{f}_{x}\right)$, and $\Lambda_{p}$ is the order of $K\left(G_{s}\right)_{p}$ such that $\left[\Lambda_{p}: Z_{p}[\tilde{X}]\right]=p^{\rho}$ for $\rho=v_{p}(f)$.

(2) If $L$ is not a square, then $t_{h}=0$. If $L$ is a square, then one has

$$
\begin{aligned}
t_{h}=- & \sum_{d} \frac{d^{\kappa-1}}{n / d-d}\left(L F_{2}^{4}\right)^{1-\kappa / 2} \sum_{f} \prod_{p \mid M_{1} M_{2} M_{4}} c_{p}^{\prime}\left(\sqrt{L} F_{2}^{2}(d+n / d), f\right) \\
& \times \varphi\left(\sqrt{L} F_{1}(n / d-d) / f\right),
\end{aligned}
$$

where $d$ runs through all positive integers such that $0<d<\sqrt{n}, d \mid n$, and $d+$ $n / d \equiv 0\left(\bmod \sqrt{L} F_{2}^{-1}\right)$, and $f$ runs through all positive integers which satisfy $f \mid(n / d-d)$ and $\left(f, \mathfrak{f}_{x} L\right)=1 . \quad c_{p}^{\prime}\left(\sqrt{L} F_{2}^{2}(d+n / d), f\right)$ is the same as in (1) for $s=\sqrt{L} F_{2}^{2}(d+n / d)$. 
(3) $t_{p}$ does not vanish only if $M_{2}=F_{2}^{2}, M_{3}=1$ or 4 , and $M_{1}$ and $n$ are squares. When this condition is satisfied,

$$
t_{p}=-\frac{n^{(\kappa-1) / 2}}{2} \mathfrak{f}_{\chi} \prod_{p \mid M_{1} M_{2}}\left(1-\frac{1}{p}\right) \prod_{p \mid M_{1} M_{2} M_{4}} c_{p}^{\prime},
$$

where $c_{p}^{\prime}=c_{p}^{\prime}\left(2 \sqrt{L} F_{2}^{2} \sqrt{n}, 1\right)$.

\section{§3. A decomposition of $S_{k}(N, \psi)$}

Let $\chi$ be a character modulo $N$, and $\chi_{0}$ the primitive character associated with $\chi$. For $\chi$, we define

$$
U_{x}=U_{\chi_{0}}, g(\chi)=g\left(\chi_{0}\right) .
$$

For characters $\chi$ and $\chi^{\prime}$ with prime power conductors, we have

TheOREM 3.1. For positive integers $N$ and $\kappa$, let $\psi$ be a character modulo $N$ such that $\psi(-1)=(-1)^{x}$. Let $p$ be a prime divisor of $N$, and $\chi, \chi^{\prime}$ characters with $\mathfrak{F}_{x}=p^{\mu}, \mathfrak{F}_{x^{\prime}}=p^{\mu^{\prime}}$ which satisfy the condition (1.1). Suppose $\mu \leq$ $\left[v_{p}(N) / 3\right], \quad \mu^{\prime} \leq\left[v_{p}(N) / 3\right]$, and $v_{p}\left(\mathfrak{f}_{\psi}\right) \leq\left[v_{p}(N) / 3\right]$. Then for $f \in S_{\kappa}^{0}(N, \psi)$, it holds

$$
\begin{array}{ll}
f\left|U_{\chi} U_{\chi^{\prime}}=\psi_{P}(-1) \bar{\psi}_{N / P}(P) f\right| U_{\chi x^{\prime}} & \text { if } \chi \neq \bar{\chi}^{\prime} \\
f \mid U_{\chi} U_{x^{\prime}}=\psi_{P}(-1)^{2} \bar{\psi}_{N / P}(P)^{2} f & \text { if } \chi=\bar{\chi}^{\prime},
\end{array}
$$

where $P=p^{\nu}$ for $\nu=v_{p}(N)$.

Proof. We may assume $\chi$ and $\chi^{\prime}$ are primitive. For integers $i, j, i^{\prime}$, and $j^{\prime}$, put

$$
\alpha_{i j}=\left(\begin{array}{cc}
\tilde{f}_{x} & i \\
0 & \mathfrak{f}_{x}
\end{array}\right) \eta_{P}\left(\begin{array}{cc}
\tilde{f}_{x} & j \\
0 & \mathfrak{f}_{x}
\end{array}\right) \eta_{P}, \quad \alpha_{i^{\prime} j^{\prime}}^{\prime}=\left(\begin{array}{ll}
\mathfrak{f}_{x^{\prime}} & i^{\prime} \\
0 & \tilde{\mathfrak{f}}_{x^{\prime}}
\end{array}\right) \eta_{P}\left(\begin{array}{ll}
\tilde{\mathfrak{f}}_{x^{\prime}} & j^{\prime} \\
0 & \tilde{\mathfrak{f}}_{x^{\prime}}
\end{array}\right) \eta_{P}
$$

Then by the definition of $U_{x}$ and $U_{x^{\prime}}$, we have

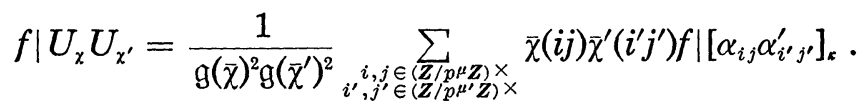

Since $f\left|U_{x} U_{x^{\prime}}=f\right| U_{x^{\prime}} U_{x}$ for $f \in S_{x}^{0}(N, \psi)$ by (1) of Prop. 1.4, we may assume $\mu \geq \mu^{\prime}$.

Case I. First we assume $\mu>\mu^{\prime}$. Let $\alpha_{i j} \alpha_{i^{\prime} j^{\prime}}^{\prime}=-p^{\nu+2 \mu^{\prime}}\left(\begin{array}{ll}A & B \\ C & D\end{array}\right)$, then by the assumption on $\mathfrak{f}_{x}, \mathfrak{f}_{x^{\prime}}$ and $\mathfrak{f}_{\psi}$, we have

$$
A \equiv-p^{\nu+2 \mu}+i_{0} j_{0} p^{2 \nu}\left(\bmod p^{\nu+3 \mu}\right)
$$




$$
\begin{aligned}
& B \equiv-i_{0} p^{\nu+\mu}\left(\bmod p^{\nu+2 \mu}\right) \\
& C \equiv j_{0} p^{2 \nu+\mu}\left(\bmod p^{2 \nu+2 \mu}\right) \\
& D \equiv-p^{\nu+2 \mu}\left(\bmod p^{\nu+3 \mu}\right),
\end{aligned}
$$

where $i_{0}=i+p^{\mu-\mu^{\prime}} i^{\prime}$ and $j_{0}=j+p^{\mu-\mu^{\prime} j^{\prime}}$. Since $\operatorname{det} \alpha_{i j} \alpha_{i^{\prime} j^{\prime}}^{\prime}$ and $\operatorname{det} \alpha_{i_{0 j} j_{0}}$ are powers of $p$, by Lemma 2.1 we see $\beta=-p^{-\nu-2 \mu^{\prime}} \alpha_{i j} \alpha_{i^{\prime} j^{\prime}}^{\prime} \alpha_{i_{0} j_{0}}^{-1} \in \Gamma_{0}(N)$ and $\psi_{P}(\beta)$ $=1$, where $\alpha_{i_{0} j_{0}}=\left(\begin{array}{cc}p^{\mu} & i_{0} \\ 0 & p^{\mu}\end{array}\right) \eta_{P}\left(\begin{array}{cc}p^{\mu} & j_{0} \\ 0 & p^{\mu}\end{array}\right) \eta_{P}$. For the other prime divisors of $N$, we have

$$
\beta \equiv\left(\begin{array}{cc}
-P & * \\
0 & -P^{-1}
\end{array}\right) \quad \bmod (N / P)^{4}
$$

Hence we obtain

$$
f\left|\left[\alpha_{i j} \alpha_{i^{\prime} j^{\prime}}^{\prime}\right]_{\kappa}=(-1)^{\kappa} \bar{\psi}_{N / P}(-P) f\right|\left[\alpha_{i_{0} j_{0}}\right]_{\kappa} .
$$

Since $\psi(-1)=(-1)^{x}$, we see

$$
\begin{aligned}
& f \mid U_{\chi} U_{x^{\prime}}=\frac{\psi_{P}(-1) \bar{\psi}_{N / P}(P)}{g(\bar{\chi})^{2} g\left(\bar{\chi}^{\prime}\right)^{2}} \sum_{\substack{i_{0}, j_{0} \bmod p^{\mu} \\
i^{\prime}, j^{\prime} \bmod p^{\prime \prime}}} \bar{\chi}\left(( i _ { 0 } - p ^ { \mu - \mu ^ { \prime } } i ^ { \prime } ) \left(j_{0}-p^{\left.\left.\mu-\mu^{\prime} j^{\prime}\right)\right)}\right.\right. \\
& \times \bar{\chi}^{\prime}\left(i^{\prime} j^{\prime}\right) f \mid\left[\alpha_{i_{0} j_{0}}\right]_{\kappa}
\end{aligned}
$$

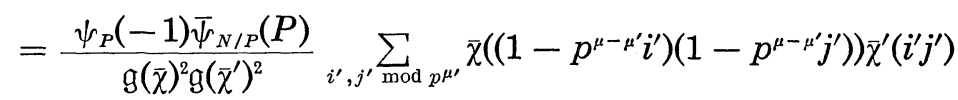

$$
\begin{aligned}
& \times \sum_{i_{0}, j_{0} \bmod p^{\mu}} \bar{\chi} \bar{\chi}^{\prime}\left(i_{0} j_{0}\right) f \mid\left[\alpha_{i_{0} j_{0}}\right]_{\kappa} \text {. }
\end{aligned}
$$

We note (c.f. Shimura [16, Lemma 8])

$$
\frac{1}{\mathfrak{g}(\bar{\chi}) \mathfrak{g}\left(\bar{\chi}^{\prime}\right)} \sum_{i^{\prime} \bmod p^{\prime \prime}} \bar{\chi}\left(1-p^{\mu-\mu^{\prime}} i^{\prime}\right) \bar{\chi}^{\prime}\left(i^{\prime}\right)=\frac{1}{\mathfrak{g}\left(\bar{\chi} \bar{\chi}^{\prime}\right)} .
$$

Thus we obtain

$$
f\left|U_{x} U_{x^{\prime}}=\psi_{P}(-1) \bar{\psi}_{N / P}(P) f\right| U_{x x^{\prime}} \cdot
$$

Case II. Next we assume $\mathfrak{f}_{x}=\mathfrak{f}_{x^{\prime}}=\mathfrak{f}_{x x^{\prime}}$. In the same way as above, we obtain

$$
f\left|\left[\alpha_{i j} \alpha_{i^{\prime} j^{\prime}}\right]_{\kappa}=\psi_{P}(-1) \bar{\psi}_{N / P}(P) f\right|\left[\alpha_{i_{0 j} j_{0}}\right]_{\kappa},
$$

where $i_{0}=i+i^{\prime}$ and $j_{0}=j+j^{\prime}$. We note $\alpha_{i_{0} j_{0}} \in \Xi\left(U_{x} T_{1}\right) \cap G L_{2}(Q)$ if and only if $i_{0}$ and $j_{0}$ are prime to $p$. Taking notice of (c.f. ibid.)

$$
\frac{1}{g(\bar{\chi}) g\left(\bar{\chi}^{\prime}\right)} \sum_{i^{\prime} \bmod p^{\mu}} \bar{\chi}\left(1-i^{\prime}\right) \bar{\chi}^{\prime}\left(i^{\prime}\right)=\frac{1}{g\left(\bar{\chi} \bar{\chi}^{\prime}\right)} ;
$$

we have 


$$
f\left|U_{\chi} U_{x^{\prime}}=\psi_{P}(-1) \bar{\psi}_{N / P}(P) f\right| U_{z x^{\prime}}+S_{1}+S_{2}+S_{3}
$$

where

$$
S_{k}=\frac{\psi_{P}(-1) \bar{\psi}_{N / P}(P)}{g(\bar{\chi})^{2} g\left(\bar{\chi}^{\prime}\right)^{2}} \sum \bar{\chi}\left(\left(i_{0}-i^{\prime}\right)\left(j_{0}-j^{\prime}\right)\right) \bar{\chi}^{\prime}\left(i^{\prime}\right) \bar{\chi}^{\prime}\left(j^{\prime}\right) f \mid\left[\alpha_{i_{0} j_{0}}\right]_{\kappa} .
$$

Here the summation is extended over $i_{0}, j_{0}, i^{\prime}, j^{\prime}$ modulo $p^{\mu}$ which satisfy the condition $(1) i_{0} \not \equiv 0(\bmod p), j_{0} \equiv 0(\bmod p),(2) i_{0} \equiv 0(\bmod p), j_{0} \not \equiv 0$ $(\bmod p)$ or $(3) i_{0} \equiv 0(\bmod p), j_{0} \equiv(\bmod p)$ according as $k=1,2$, or 3 . We show $S_{1}=S_{2}=S_{3}=0$. Let $f=\sum_{m \geq 1} a_{m} e^{2 \pi i m z}$ be the Fourier expansion of $f$. In the case of $S_{1}$, put $i_{0}=p u$. Then we see

$$
\begin{aligned}
& \sum_{\substack{i^{\prime} \bmod p^{\mu} \\
u^{\prime} \bmod p^{\mu-1}}} \bar{\chi}\left(p u-i^{\prime}\right) \bar{\chi}^{\prime}\left(i^{\prime}\right) f \mid\left[\left(\begin{array}{cc}
P & p u \\
0 & P
\end{array}\right)\right]_{x} \\
& \quad=\sum_{m} a_{m} \sum_{u, i^{\prime}} \bar{\chi}\left(p u-i^{\prime}\right) \bar{\chi}^{\prime}\left(i^{\prime}\right) e^{2 \pi i p u m / p \mu} e^{2 \pi i m z} \\
& \quad=\sum_{m} a_{m} \sum_{u} \bar{\chi}(p u-1) \sum_{\left(i^{\prime}, p\right)=1} \bar{\chi} \bar{\chi}^{\prime}\left(i^{\prime}\right) e^{2 \pi i p u m / p \mu} e^{2 \pi i m z} \\
& \quad=0
\end{aligned}
$$

since the conductor of $\chi \chi^{\prime}$ is $p^{\mu}$. This shows $S_{1}=0$. We can treat the cases of $S_{2}$ and $S_{3}$ in the same way, and we omit the proof.

Case III. Finally we assume $\mathfrak{f}_{x}=\mathfrak{f}_{\chi^{\prime}}>\mathfrak{f}_{x x^{\prime}}$. Put $\chi^{\prime \prime}=\chi \chi^{\prime}$, then $\chi^{\prime}=\bar{\chi} \chi^{\prime \prime}$. By Case I, we have $U_{x^{\prime}}=\psi_{P}(-1) \psi_{N / P}(P) U_{\bar{\chi}} U_{\chi^{\prime \prime}}$. If we prove $U_{\chi} U_{\bar{\chi}}$ $=\left(\psi_{P}(-1) \psi_{N / P}(P)\right)^{2}$, we obtain $U_{\chi} U_{\chi^{\prime}}=\psi_{P}(-1) \psi_{N / P}(P) U_{\chi} U_{\bar{z}} U_{\chi^{\prime \prime}}=$ $\psi_{P}(-1) \bar{\psi}_{N / P}(P) U_{\chi^{\prime \prime}}$. Hence it is enough to show $U_{x} U_{\bar{\chi}}=\left(\psi_{P}(-1) \psi_{N / P}(P)\right)^{2}$, and we may assume $\chi^{\prime}=\bar{\chi}$. As in the case II, we have

$$
f \mid U_{\chi} U_{\bar{\chi}}=\frac{\psi_{P}(-1) \bar{\psi}_{N / P}(P)}{(g(\bar{\chi}) g(\chi))^{2}}\left(T_{1}+T_{2}+T_{3}+T_{4}\right),
$$

where

$$
T_{k}=\sum \chi\left(\left(i_{0}-i^{\prime}\right)\left(j_{0}-j^{\prime}\right)\right) \chi\left(i^{\prime} j^{\prime}\right) f \mid\left[\alpha_{i_{0} j_{0}}\right]_{\kappa} .
$$

Here the summation is extended over $i_{0}, j_{0}, i^{\prime}, j^{\prime}$ modulo $p^{\mu}$ which satisfy the condition $(1) i_{0} \not \equiv 0(\bmod p), j_{0} \not \equiv 0(\bmod p)(2) i_{0} \not \equiv 0(\bmod p), j_{0} \equiv 0$ $(\bmod p),(3) i_{0} \equiv 0(\bmod p), j_{0} \neq \equiv 0(\bmod p)$, or (4) $i_{0} \equiv 0(\bmod p), j_{0} \equiv 0$ $(\bmod p)$ according as $k=1,2,3$, or 4 . Let $f=\sum_{m \geq 1} a_{m} e^{2 \pi i m z}$ be the Fourier expansion of $f$, then $a_{m}=0$ if $p$ divides $m$. We see

$$
T_{1}=\left(\sum_{i^{\prime}} \chi\left(1-i^{\prime}\right) \chi\left(i^{\prime}\right)\right)^{2} \sum_{\substack{\left(i_{0}, p\right)=1 \\\left(j_{0}, p\right)=1}} f \mid\left[\alpha_{i_{0} j_{0}}\right]_{\kappa}
$$


and

$$
\begin{aligned}
& \sum_{i_{0} \in\left(Z / p^{\mu} Z\right) \times} f \mid\left[\left(\begin{array}{cc}
p^{\mu} & i_{0} \\
0 & p^{\mu}
\end{array}\right)\right]_{\kappa}=\left\{\begin{array}{cl}
-f & \text { if } \mu=1 \\
0 & \text { otherwise },
\end{array}\right. \\
& \sum_{i^{\prime} \bmod p^{\mu}} \chi\left(1-i^{\prime}\right) \bar{\chi}\left(i^{\prime}\right)=-\chi(-1) \quad \text { if } \mu=1 \text {. }
\end{aligned}
$$

From this we obtain

$$
T_{1}=\left\{\begin{array}{cl}
f \mid\left[\eta_{P}^{2}\right]_{\kappa} & \text { if } \mu=1 \\
0 & \text { otherwise }
\end{array}\right.
$$

In the similar way, we can verify

$$
\begin{aligned}
T_{2}=T_{3} & =\left\{\begin{array}{cl}
(p-1) f \mid\left[\eta_{P}^{2}\right]_{\kappa} & \text { if } \mu=1 \\
0 & \text { otherwise }
\end{array}\right. \\
T_{4} & = \begin{cases}(p-1)^{2} f \mid\left[\eta_{P}^{2}\right]_{\varsigma} & \text { if } \mu=1 \\
p^{2 \mu} f \mid\left[\eta_{P}^{2}\right]_{\varsigma} & \text { otherwise }\end{cases}
\end{aligned}
$$

Our assertion follows from this and Lemma 1.1. This completes the proof.

By the above theorem and Cor. 1.3, we obtain

CoRollary 3.2. Let $\chi$ and $\chi^{\prime}$ be the characters which satisfy (1.1). Suppose $v_{p}\left(\mathfrak{F}_{\chi}\right) \leq v_{p}(N) / 3, v_{p}\left(\mathfrak{F}_{\chi^{\prime}}\right) \leq v_{p}(N) / 3$, and $v_{p}\left(\mathfrak{F}_{x}\right) \leq v_{p}(N) / 3$ for each prime divisor $p$ of $\mathrm{f}_{\chi} \mathrm{f}_{\chi^{\prime}}$. Then for $f \in S_{\star}^{0}(N, \psi)$, it holds

$$
f\left|\tilde{U}_{\chi} \tilde{U}_{x^{\prime}}=f\right| \tilde{U}_{\chi x^{\prime}}
$$

Let $M$ be a divisor of $N$ such that $M^{3} \mid N$, and assume $3 v_{p}\left(\mathfrak{f}_{\psi}\right) \leq v_{p}(N)$ for any pirme divisor $p$ of $M$. Let $X(M)$ be the group of all characters defined modulo $M$, and $\tilde{U}$ the group consisting of operators $\tilde{U}_{x}$ acting on $S_{x}^{0}(N, \psi)$ for $X(M)$. Then Cor. 3.2 says that the map $\mathfrak{U}: \chi \rightarrow \tilde{U}_{z}$ gives a homomorphism from $X(M)$ to $\tilde{U}$. By means of this homomorphism, we can decompose $S_{\kappa}^{0}(N, \psi)$ as follows;

$$
S_{\kappa}^{0}(N, \psi)=\bigoplus_{a \in(Z / M Z) \times} S_{\kappa}^{0}(N, \psi, a),
$$

where

$$
S_{\kappa}^{0}(N, \psi, a)=\left\{f \in S_{\kappa}^{0}(N, \psi)|f| \tilde{U}_{\chi}=\chi(a) f \quad \text { for } \chi \in X(M)\right\} .
$$

On these subspace, the Hecke operator $T_{n}$ acts and the trace of $T_{n}$ on them are given by

$$
\operatorname{tr} T_{n}\left|S_{\star}^{0}(N, \psi, a)=\frac{1}{\left|(Z \mid M Z)^{\times}\right|} \sum_{x \in X(M)} \bar{\chi}(a) \operatorname{tr} \tilde{U}_{x} T_{n}\right| S_{\star}^{0}(N, \psi)
$$


the trace $\operatorname{tr} \tilde{U}_{z} T_{n} \mid S_{x}^{0}(N, \psi)$ are given by Hijikata [8] for the trivial $\chi$ and by Th. 2.5 in this paper for general $\chi$. In the case where $\psi$ is the trivial character, we can consider also the action of $W_{L}$ to decompose $S_{\kappa}(N)$. Let $\tilde{W}$ denote the group of all $W_{L}$ for $L \mid N$, and $E(W)$ the character group of $\tilde{W}$. We define $S_{\kappa}^{0}(N, a, e)$ for $a \in(Z / M Z)^{\times}$and $e \in E(W)$ by

$$
\begin{aligned}
S_{\kappa}^{0}(N, a, e)=\left\{f \in S_{\kappa}^{0}(N)|f| \tilde{U}_{x}=\chi(a) f\right. & \text { for } \chi \in X(M), \\
f \mid W_{L}=e\left(W_{L}\right) f & \text { for } \left.W_{L} \in E(W)\right\} .
\end{aligned}
$$

Then we have

$$
S_{\star}^{0}(N)=\underset{a \in(Z / M Z) \times}{\bigoplus} \underset{e \in E(W)}{\oplus} S_{\kappa}^{0}(N, a, e),
$$

and the trace of $T_{n}$ on $S_{\kappa}^{0}(N, a, e)$ is expressed as follows;

$$
\operatorname{tr} T_{n}\left|S_{x}^{0}(N, a, e)=\frac{1}{\left|(Z \mid M Z)^{\times}\right||E(W)|} \sum_{\substack{x \in X(M) \\ W \in W}} \bar{\chi}(a) \bar{e}(W) \operatorname{tr} \tilde{U}_{\chi} W_{L} T_{n}\right| S_{x}^{0}(N) .
$$

a formula for $\operatorname{tr} U_{x} W T_{n}$ is given by Yamauchi [18] for the trivial $\chi$ and by Th. 2.9 for the general $\chi$.

Now we take $N=p^{\nu}$ with a prime $p$ and a positive integer $\nu \geq 3$ and $\psi$ the trivial character. Under such a condition, we have given in [9] a decomposition of $S_{k}^{0}\left(p^{\nu}\right)$ into four subspaces $S_{\mathrm{I}}, S_{\mathrm{II}}, S_{\mathrm{II}}, S_{\mathrm{III}}$. We compare this decomposition with that given above. Put $M=p^{[\nu / 3]}$. Then for example, the subspace $S_{\mathrm{I}}$ is defined by

$$
S_{\mathrm{I}}=\left\{f \in S_{s}^{0}(N)|f| U_{s}=f, f \mid W_{N}=f\right\},
$$

where $\varepsilon$ is the quadratic residue symbol modulo $p$. This space is expressed by our spaces $S_{\star}^{0}(N, a, e)$ as follows;

$$
S_{\mathrm{I}}=\underset{\substack{a \in(Z M M Z) \times \\ s(a)=1}}{\bigoplus} S^{0}(N, a, 1)
$$

where 1 denotes the trivial character of $\tilde{W}$. This shows that even in the case where $\nu=3$ our decomposition of $S_{k}^{0}(N)$ gives a finer one that in [11]. In the next section, we give a numerical example in the case where $p=$ $11, \kappa=2$, and $\nu=3$.

We prove two more properties of $U_{x}$.

Proposition 3.3. The notation being as above, let $f$ be a primitive form in $S_{k}^{0}(N, \psi)$. For a character $\chi$ with $\mathfrak{f}_{x}=p^{\mu}$ which satisfies (1.1), let $f \mid \tilde{U}_{x}$ $=c_{\chi} f$. For $\sigma \in \operatorname{Gal}(\overline{\mathbf{Q}} / \mathbf{Q})$ and $\zeta=e^{2 \pi i / p^{\mu}}$, let $\zeta^{\sigma}=\zeta^{n}$ with $n \in Z$, and for $f=$ $\sum_{m \geq 1} a_{m} e^{2 \pi i m z}$, put $f^{o}=\sum_{m \geq 1} a_{m}^{o} e^{2 \pi i m z}$. Then it holds 


$$
f^{\sigma} \mid \tilde{U}_{\chi} \sigma=\chi\left(n^{2}\right)^{\sigma}\left(\sqrt{p^{\sigma}} / \sqrt{p}\right)^{x} c_{\chi}^{\sigma} f^{\sigma} .
$$

Proof. Let $G_{+}=\left\{x \in G L_{2}\left(\boldsymbol{Q}_{A}\right) \mid \operatorname{det} x_{\infty}>0\right\}$, and $\boldsymbol{Q}_{a b}$ the maximal abelian extension of $\boldsymbol{Q}$. Let $\rho$ be a homomorphism of $\boldsymbol{G}_{+}$onto $\mathrm{Gal}\left(\boldsymbol{Q}_{a b} / \boldsymbol{Q}\right)$ obtained by defining $\rho(x)$ to be the action of $(\operatorname{det} x)^{-1}$ on $\boldsymbol{Q}_{a b}$. Let $G$ be a subgroup of $G_{+} \times \operatorname{Gal}(\overline{\boldsymbol{Q}} / \boldsymbol{Q})$ given by

$$
G=\left\{(x, \sigma) \in G_{+} \times \operatorname{Gal}(\overline{\mathbf{Q}} / \boldsymbol{Q}) \mid \rho(x)=\sigma \text { on } \boldsymbol{Q}_{a b}\right\} .
$$

Then Shimura [17, Th. 1.5] defined an action of $G$ on modular forms. We denote the action of $(x, \sigma)$ by $f^{(x, \sigma)}$. Let $t$ be an element of $\prod_{p} Z_{p}^{\times}$such that $\rho(x)=\sigma$ on $\boldsymbol{Q}_{a b}$ for $x=\left(\begin{array}{ll}1 & 0 \\ 0 & t\end{array}\right)$. Let $\alpha_{i j}$ and $\tilde{\chi}$ be the same as in the proof of Th. 3.1, and consider the action of $(x, \sigma)$ on the both sides of

$$
\frac{\psi_{P}(-1) \bar{\psi}_{N / P}(P) \chi(N / P)}{g(\bar{\chi})^{2}} \sum_{i, j} \tilde{\chi}\left(\alpha_{i j}\right) f \mid\left[\alpha_{i j}\right]_{\kappa}=c_{\chi} f,
$$

where $P=p^{\nu}$. Then the right hand side becomes $c_{\chi}^{\sigma} f^{\sigma}$. Observe that $\left(g(\bar{\chi})^{2}\right)^{\sigma}$ $=\chi\left(n^{2}\right)^{\sigma} g\left(\bar{\chi}^{\sigma}\right)^{2}$ and $f^{\left(\alpha_{i j}, 1\right)(x, o)}=\left(f^{\sigma}\right)^{\left(x-1_{\alpha_{i j}}, 1\right)}$. Choose $t_{0} \in Z$ such that $t_{0} \equiv t_{q}$ $\left(\bmod q^{4}\right)$ for each prime $q \mid N$. Let $i^{\prime}$ and $j^{\prime}$ be integers such that $i^{\prime} \equiv t_{0} i$ $\left(\bmod P^{4}\right)$ and $t_{0} j^{\prime} \equiv j\left(\bmod P^{4}\right)$, and let $A$ be an integer such that $A$ $\equiv p^{\nu-\mu}\left(-t_{0} j+j^{\prime}\right)\left(\bmod (N / P)^{4}\right)$ and $A \equiv 0\left(\bmod P^{4}\right)$. Then we see

$$
x^{-1} \alpha_{i j} x \equiv\left(\begin{array}{cc}
1 & A \\
0 & 1
\end{array}\right) \alpha_{i^{\prime} j^{\prime}} \quad\left(\bmod N^{4}\right) \text {. }
$$

Hence $f^{\left(\alpha_{i j}, 1\right)(x, \sigma)}=\left(f^{o}\right)^{\left(\alpha_{i^{\prime} j^{\prime}}, 1\right)}$, and we obtain

$$
\left(f \mid\left[\alpha_{i j}\right]_{k}\right)^{(x, o)}=\left(\sqrt{p^{\sigma}} / \sqrt{p}\right)^{x} f^{\sigma} \mid\left[\alpha_{i^{\prime} j^{\prime}}\right]_{\kappa} .
$$

Noting $\chi\left(\alpha_{i j}\right)=\chi\left(\alpha_{i^{\prime} j^{\prime}}\right)$, we obtain

$$
\frac{\psi_{P}^{\sigma}(-1) \bar{\psi}_{N / P}^{\sigma}(P) \chi^{\sigma}(N / P)}{\mathfrak{g}\left(\bar{\chi}^{\sigma}\right)^{2}} \sum_{i, j} \tilde{\chi}^{\sigma}\left(\alpha_{i j}\right) f^{\sigma} \mid\left[\alpha_{i j}\right]_{\kappa}=\chi\left(n^{2}\right)^{\sigma}(\sqrt{p} \sigma / \sqrt{p})^{\kappa} c_{\chi}^{\sigma} f^{\sigma} .
$$

Since $f \in S_{\kappa}^{0}\left(N, \psi^{\sigma}\right)$, this prove our proposition.

Corollary 3.4. Let $f$ be a primitive form in $S_{x}^{0}(N, \psi)$, and $K_{f}$ the field generated by all the Fourier coefficients $a_{m}$ of $f$ over $\boldsymbol{Q}$. Suppose $v_{p}\left(\mathfrak{f}_{\psi}\right) \leq$ $v_{p}(N) / 3$ and $\mu=\left[v_{p}(N) / 3\right] \geq 1$ for a prime divisor $p$ of $N$. Then $K_{f}$ contains $F_{p^{\mu}}=Q\left(e^{2 \pi i / p^{\mu}}+e^{-2 \pi i / p^{\mu}}\right)$ (resp. $\left.F_{p^{\mu-1}}\right)$ if $\kappa$ is even and $p$ is odd (resp. $p=2)$, and $K_{f}(\sqrt{p})$ contains $F_{p^{\mu}}\left(\right.$ resp. $\left.F_{p^{\mu-1}}\right)$ if $\kappa$ is odd and $p$ is odd (resp. $p=2$ ).

Proof. We prove only the case where $\kappa$ is even and $p$ is odd. The other case can be treated in a similar way. In this case, it is enough to 
prove that for $\sigma \in \operatorname{Gal}(\bar{Q} / Q) \sigma \mid F_{p} \mu=$ the identity if $\sigma \mid K_{f}=$ the identity. Assume $\sigma \mid K_{f}$ is the identity, then $f^{\sigma}=f$ and $\psi^{\sigma}=\psi$. In the above notation, we may assume $f \in S_{\star}^{0}(N, \psi, a)$ for some $a$. Then $c_{\chi}=\chi(a)$ for $\chi \epsilon$ $X\left(p^{\mu}\right)$. From this and the above proposition, it follows

$$
\chi(a)^{\sigma}=\chi\left(n^{2}\right)^{\sigma} \chi(a)^{\sigma}\left(\sqrt{p^{\sigma}} / \sqrt{p}\right)^{x},
$$

for all $\chi \in X\left(p^{\mu}\right)$, where $n$ is an integer such that $\left(e^{2 \pi i / p^{\mu}}\right)^{\sigma}=e^{2 \pi i n / p^{\mu}}$. Since $\kappa$ is even, $\chi\left(n^{2}\right)=1$ for all $\chi \in X\left(p^{\mu}\right)$, and $n^{2} \equiv 1\left(\bmod p^{\mu}\right)$. If $p$ is odd, this implies $n= \pm 1\left(\bmod p^{\mu}\right)$ hence $\sigma \mid F_{p^{\mu}}=$ the identity. This proves our corollary.

Proposition 3.5. The notation being as in Prop. 3.3, assume $\nu-2 \mu$ $>0$ and $v_{p}\left(\mathfrak{f}_{\psi}\right)<\nu-2 \mu$ for $\nu=v_{p}(N)$ and $\mu=v_{p}\left(\mathfrak{f}_{x}\right)$. Then it holds

$$
f\left|U_{\chi} W_{P}=f\right| W_{P} U_{x},
$$

where $P=p^{\nu}$.

Proof. First we note $\eta_{P}$ normalizes the set $\Xi\left(U_{x} T_{1}\right) \cap G L_{2}(Q)$. For $g=\left(\begin{array}{ll}a & b \\ c & d\end{array}\right) \in \Xi\left(U_{x} T_{1}\right) \cap G L_{2}(Q)$, we note

$$
\eta_{P}^{-1} g \eta_{P}=\left\{\begin{array}{cc}
\left(\begin{array}{cc}
d & -c / p^{\nu} \\
-b p^{\nu} & a
\end{array}\right) & \left(\bmod P^{3}\right) \\
\left(\begin{array}{cc}
a & b / p^{\nu} \\
c p^{\nu} & d
\end{array}\right) & \left(\bmod (N / P)^{4}\right),
\end{array}\right.
$$

and $\bar{\psi}_{p}\left(-d / p^{\nu+2 \mu}\right)=\psi_{p}\left(-a / p^{\nu+2 \mu}\right)$ by the assumption on $\psi$. Our assertion follows from this and Cor. 2.3.

\section{§4. Numerical examples and a congruence between cusp forms}

We shall gives examples of characteristic polynomials of Hecke operators taking $N=11^{3}, \kappa=2$ and $\psi=$ the trivial character and discuss a congruence property between cusp forms. We use the notation in $\S 3$. Let $S_{\text {III }}$ be the subspace of $S_{*}^{0}\left(p^{\nu}\right)$ given by

$$
S_{\mathrm{III}}=\left\{f \in S_{\varepsilon}^{0}(N)|f| U_{\varepsilon}=f, f \mid W_{P}=-f\right\},
$$

where $\varepsilon$ is the quadratic residue symbol modulo $p$ and $P=p^{\nu}$. In our case, we find $\operatorname{dim} S_{\mathrm{I}}=15$ and $\operatorname{dim} S_{\mathrm{III}}=35$. By means of the decomposition introduced in $\S 3$, these subspaces can be written as follows;

$$
S_{\mathrm{I}}=\underset{\substack{a \text { mod } 11 \\ \varepsilon(a)=1}}{\bigoplus} S_{2}\left(11^{3}, a, 1\right), \quad S_{\mathrm{III}}=\underset{\substack{a \text { mod 11 } \\ \delta(a)=1}}{\bigoplus} S_{2}\left(11^{3}, a,-1\right),
$$


where -1 denotes the non-trivial homomorphism from $\left\{W_{P}, 1\right\}$ to $\{ \pm 1\}$. For $a$ such that $\varepsilon(a)=1$, we find $\operatorname{dim} S_{2}\left(11^{3}, a, 1\right)=3$ and $\operatorname{dim} S_{2}\left(11^{3}, a,-1\right)$ $=7$. Taking $a=4$, we give characteristic polynomial of Hecke operator $T_{n}$ acting on these subspace for some $n$.

\begin{tabular}{r|r|c|l|c}
$n$ & $\varepsilon(n)$ & \multicolumn{1}{|c|}{$a_{n}$} & \multicolumn{1}{|c|}{$f_{T_{n}}(X)$} & $N\left(f_{T_{n}}\left(a_{n}\right)\right)$ \\
\hline 2 & -1 & 0 & $X^{2}+\alpha^{3}-3 \alpha-3$ & 199 \\
3 & 1 & $-\alpha^{4}-2 \alpha^{3}+3 \alpha^{2}+5 \alpha-2$ & $\left(X-\alpha^{3}+3 \alpha\right)^{2}$ & $199^{2}$ \\
5 & 1 & $-\alpha^{4}+5 \alpha^{2}-\alpha-5$ & $(X-\alpha+1)^{2}$ & $199^{2}$ \\
199 & 1 & $-6 \alpha^{4}-13 \alpha^{3}+30 \alpha^{2}$ & $\left(X-4 \alpha^{4}+8 \alpha^{3}+13 \alpha^{2}\right.$ & $(11 \cdot 23 \cdot 43 \cdot 199)^{2}$ \\
& & $+39 \alpha-18$ & $-16 \alpha+11)^{2}$ &
\end{tabular}

Here $\alpha=e^{2 \pi i / 11}+e^{-2 \pi i / 11}$ and $N$ denotes the norm from $F_{11}=\boldsymbol{Q}(\alpha)$ to $\boldsymbol{Q}$. For an explanation of the table, we remark that $S_{2}\left(11^{3}, 4,1\right)$ contains a primitive form $\theta_{\mathrm{I}}$ associated with a Grössencharacter of $Q(\sqrt{-11}) . \quad a_{n}$ denotes the $n$-th Fourier coefficient of $\theta_{\mathrm{I}}$, that is, the eigenvalue for $T_{n} . f_{T_{n}}(X)$ denotes the characteristic polynomial for $T_{n}$ on the orthogonal complement $S_{\mathrm{I}}^{0}$ of the one dimensional subspace spanned by $\theta_{\mathrm{I}}$. We note $N\left(f_{T_{n}}\left(a_{n}\right)\right)$ is divided by the prime 199 in our table and this suggest a congruence between $\theta_{\mathrm{I}}$ and a primitive form $f \in S_{\mathrm{I}}^{0}$ modulo a prime ideal $\mathfrak{p}$ in $K_{f}$ which divides 199. In fact, Prop. 4.2 in [11] implies such a congruence, and this proposition has been proved as an application of the Shimura's theory on the construction of class fields over real quadratic fields [15].

Now we take $S_{2}\left(11^{3}, 4,-1\right)$. This space also contains a primitive form $\theta_{\text {III }}$ associated with a Grössencharacter of $Q(\sqrt{-11})$. Let $b_{n}$ be the $n$-th Fourier coefficients of $\theta_{\text {III }}$, and $S_{\text {III }}^{0}$ the orthogonal complement of the one dimensional subspace spanned by $\theta_{\mathrm{III}}$. We denote by $g_{T_{n}}(X)$ the characteristic polynomial of $T_{n}$ on $S_{\mathrm{III}}^{0}$.

\begin{tabular}{|c|c|c|}
\hline$n$ & $\varepsilon(n)$ & $g_{T_{n}}(X)$ \\
\hline 2 & -1 & $\begin{aligned} X^{6}-\left(\alpha^{3}-3 \alpha+12\right) X^{4}+\left(-2 \alpha^{4}+7 \alpha^{3}+8 \alpha^{2}-21 \alpha+35\right) X^{2} \\
-\left(-14 \alpha^{4}+4 \alpha^{3}+56 \alpha^{2}-18 \alpha-4\right)\end{aligned}$ \\
\hline 3 & 1 & $\begin{array}{r}\left(X^{3}-\left(-\alpha^{4}-\alpha^{3}+3 \alpha^{2}+3 \alpha\right) X^{2}+\left(-\alpha^{4}-2 \alpha^{3}+\alpha^{2}+5 \alpha-2\right) X\right. \\
\left.-\left(2 \alpha^{4}-7 \alpha^{2}-2 \alpha+3\right)\right)^{2}\end{array}$ \\
\hline 5 & 1 & $\begin{aligned}\left(X^{3}-\left(2 \alpha^{4}-7 \alpha^{2}+4\right) X^{2}+\left(\alpha^{4}-\right.\right. & \left.\alpha^{3}-3 \alpha^{2}-5\right) X \\
& \left.-\left(-8 \alpha^{4}-5 \alpha^{3}+28 \alpha^{2}+11 \alpha-15\right)\right)^{2}\end{aligned}$ \\
\hline
\end{tabular}




\begin{tabular}{l|l|l}
$n$ & $b_{n}$ & \multicolumn{1}{|c}{$N\left(g_{7_{n}}\left(b_{n}\right)\right)$} \\
\hline 2 & 0 & $2^{2} \cdot 99527$ \\
3 & $\alpha^{4}+2 \alpha^{3}-3 \alpha^{2}-6 \alpha+2$ & $(11 \cdot 99527)^{2}$ \\
5 & $-2 \alpha^{4}+7 \alpha^{2}+\alpha-1$ & $(1429 \cdot 99527)^{2}$
\end{tabular}

Here $\alpha$ and $N$ are as above. This table also suggests a congruence between $\theta_{\text {III }}$ and a primitive form $g$ in $S_{\text {III }}^{0}$ modulo a prime ideal $\mathfrak{p}$ in $K_{g}$ which divides 99527. By virtue of the theory of Shimura, we may prove this congruence if we can compute $g_{T_{99527}}$. However, it is difficult. So we proceed in quite another way.

For positive integers $N$ and $\lambda$, let $\psi$ be a character modulo $N$ such that $\psi(-1)=(-1)^{x}$. For a prime divisor $p$ of $N$, put $\nu=v_{p}(N), \nu_{0}=[(\nu-1) / 2]$, and $M=N / p^{\nu}$. Let $\kappa^{\prime}$ and be $\kappa^{\prime \prime}$ positive integers such that $\kappa=\kappa^{\prime}+\kappa^{\prime \prime}$ and $\omega$ be a character modulo $p$ such that $\omega(-1)=(-1)^{\kappa^{\prime \prime}}$. First we prove

Lemma 4.1. The notation being as above, for a primitive form $f \in$ $S_{\boldsymbol{k}^{\prime}}^{0}(N, \psi \omega)$ and $g \in G_{\boldsymbol{k}^{\prime \prime}}(p M, \bar{\omega})$, put $F(z)=g\left(p^{\nu 0} z\right) f(z)$. Let $\chi$ be a character with $\mathfrak{f}_{\chi}=p^{\mu}$, and assume $1 \leq \mu \leq \nu_{0}$, and $v_{p}\left(\mathfrak{f}_{\psi}\right) \leq v_{p}(N) / 3$. Then $F(z)$ belongs to $S_{k}(N, \psi)$, and it holds

$$
F(z) \mid \tilde{U}_{z}=g\left(p^{\nu 0} z\right)\left(f(z) \mid \tilde{U}_{z}\right) .
$$

Proof. The first assertion is obvious. We prove the above equality. By the assumption $1 \leq \mu \leq \nu_{0}$, we have

$$
F(z) \mid R_{x}=g\left(p^{\nu 0} z\right)\left(f(z) \mid R_{x}\right) .
$$

Let $P=p^{\nu}$, then we see $g\left(p^{\nu 0} z\right) \mid W_{P}=h\left(p^{\nu 0} z\right)$ for $h \in M_{\kappa^{\prime \prime}}(p M, \omega)$, since we have

$$
\left(\begin{array}{ll}
p^{\nu_{0}} & 0 \\
0 & 1
\end{array}\right) \eta_{P} \equiv \begin{cases}p^{\nu_{0}}\left(\begin{array}{cc}
0 & -1 \\
1 & 0
\end{array}\right)\left(\begin{array}{cc}
p & 0 \\
0 & 1
\end{array}\right)\left(\begin{array}{cc}
p^{\nu-\nu_{0}-1} & 0 \\
0 & 1
\end{array}\right) & \left(\bmod P^{4}\right) \\
p^{\nu_{0}}\left(\begin{array}{ll}
p & 0 \\
0 & 1
\end{array}\right)\left(\begin{array}{cc}
p^{\nu-\nu_{0}-1} & 0 \\
0 & 1
\end{array}\right) & \left(\bmod (N / P)^{4}\right)\end{cases}
$$

and $\nu-\nu_{0}-1 \geq \nu_{0}$. Hence we obtain

$$
\begin{aligned}
F(z) \mid U_{x} & =\left(h\left(p^{\nu 0} z\right)\left(f(z) \mid R_{\chi} W_{P}\right)\right) \mid R_{\chi} W_{P} \\
& =g\left(p^{\nu 0} z\right) \mid W_{P}^{2}\left(f(z) \mid U_{\chi}\right) \\
& =\omega(-1) g\left(p^{\nu 0} z\right)\left(f(z) \mid U_{\chi}\right) .
\end{aligned}
$$

This proves our lemma. 
Corollary 4.2. The notation being as above, let $N=p^{\nu}$ with an odd prime $p$ and $\nu \geq 3$. Then $F(z)$ is contained in $S_{x}^{0}(N, \psi)$.

Proof. This follows from (2), (3) of Prop. 1.4, and the above Lemma 4.1 by taking, for example, $\chi=\varepsilon$.

We apply this Lemma taking as $f$ a primitive form associated with a Grössencharacter of $Q(\sqrt{-11})$ and as $g$ an Eisenstein series. First of all, we study the eigenvalues for $\tilde{U}_{x}$ of primitive forms associated with Grössencharacters. Let $p$ be a prime congruent to 3 modulo 4 , and a Grössencharacter of $Q(\sqrt{-p})$ which satisfies

$$
\lambda((a))=\left(\frac{a}{|a|}\right)^{u}
$$

for $a \in \boldsymbol{Q}(\sqrt{-p})$ with $a \equiv 1\left(\bmod (\sqrt{-p})^{\alpha}\right)$, where $\alpha$ is a positive integer. For $\lambda$ with $u=\kappa-1$ put

$$
\theta_{x}(z)=\sum_{a} \lambda(a) N \mathfrak{a}^{(\kappa-1) / 2} e^{2 \pi i N a z},
$$

where the summation is extended over all integral ideal of $Q(\sqrt{-p})$ prime to $(\sqrt{-p})$. Then it is known [14] that $\theta_{\lambda}$ belongs to $S_{k}(P, \psi)$ for $P=p^{\alpha+1}$ and a character $\psi$ modulo $P$ defined by

$$
\psi(a)=\lambda((a))\left(\frac{-p}{a}\right) \quad \text { for } 0 \neq a \in Z,
$$

and $\theta_{\lambda}$ is a primitive form in $S_{\kappa}^{0}(P, \psi)$ if $\lambda$ is of conductor $\left(\sqrt{-p^{\alpha}}\right)$.

Proposition 4.3. Let $\lambda$ be a Grössencharacter of $Q(\sqrt{-p})$ of conductor $\left(\sqrt{-p^{\alpha}}\right)$ for a positive integer $\alpha$, and $\chi$ a character with $\mathfrak{f}_{\chi}=p^{\mu}$. Assume $\mu \leq \alpha / 2$. Then it holds

$$
\theta_{x} \mid \tilde{U}_{x}=(g(\lambda \chi \circ N) / g(\lambda)) \theta_{\lambda},
$$

where $N$ is the norm from $Q(\sqrt{-p})$ to $\boldsymbol{Q}$, and $\mathrm{g}(\lambda \chi \circ N)$ and $\mathrm{g}(\lambda)$ are the Gauss sum of $\lambda \chi \circ N$ and $\lambda$ respectively.

Proof. For a Grössencharacter $\lambda^{\prime}$ of $\boldsymbol{Q}(\sqrt{-p})$ with the conductor $\left(\sqrt{-p^{\alpha}}\right)$, by means of the functional equation of the $L$-function of $\lambda^{\prime}$, we obtain

$$
\theta_{\lambda^{\prime}} \mid W_{P}=(\sqrt{-1})^{2 x+1} \frac{g\left(\lambda^{\prime}\right)}{p^{\alpha / 2}} \theta_{\bar{x}^{\prime}}
$$


where $P=p^{\alpha+1}$. Observe $\theta_{\lambda^{\prime}} \mid R_{z}=\theta_{\lambda^{\prime} \times \circ N}$. From this, it follows $\theta_{\lambda} \mid U_{z}=$ $-\left(g(\lambda \chi \circ N) g(\bar{\lambda}) / p^{\alpha}\right) \theta_{\lambda}$. Since $g(\lambda) g(\bar{\lambda})=(-1)^{x-1} p^{\alpha}$, we obtain

$$
\theta_{\kappa} \mid U_{\chi}=(-1)^{x}(g(\lambda \chi \circ N) / g(\lambda)) \theta_{\lambda} .
$$

Since $\psi(-1)=(-1)^{x}$, this proves the proposition.

Proposition 4.4. The notation being as in Prop. 4.3, put $c_{\lambda}(\chi)=$ $\mathfrak{g}(\lambda \chi \circ N) / \mathfrak{g}(\lambda)$. If $\eta$ is a Grössencharacter of $\boldsymbol{Q}(\sqrt{-p})$ of conductor $(\sqrt{-p})$ which satisfies (4.1) for $u=k^{\prime}-1$, then it holds

$$
c_{\lambda \eta}(\chi)=c_{\lambda}(\chi),
$$

for any character $\chi$ which satisfies $\mu \leq \alpha / 2$.

Proof. To prove this proposition, it is enough to show $\mathfrak{g}(\lambda \eta \chi \circ N) / \mathfrak{g}(\lambda \chi \circ N)$ $=g(\lambda \eta) / g(\lambda)$. Let $\mathfrak{o}$ be the ring of integers of $Q(\sqrt{-p})$, and for $a \in \mathfrak{o}$, put

$$
\lambda_{0}(a)=\lambda((a))\left(\frac{a}{|a|}\right)^{-(k-1)}, \quad \eta_{0}(a)=\eta((a))\left(\frac{a}{|a|}\right)^{-\left(k^{\prime}-1\right)} .
$$

Then we have

$$
g(\lambda \eta)=(b /|b|)^{x+\kappa^{\prime}-2} \sum_{a \in 0} \lambda_{\bmod \left(\sqrt{-p^{\alpha}}\right)} \lambda_{0} \eta_{0}(a) e^{2 \pi i \operatorname{tr}(a / b)},
$$

where $b={\sqrt{-p^{\alpha+1}}}^{\alpha}$ and $\operatorname{tr}$ denotes the trace from $\boldsymbol{Q}(\sqrt{-p})$ to $\boldsymbol{Q}$. Since

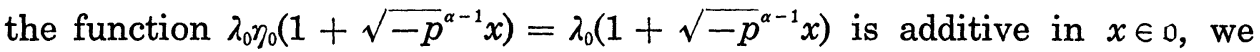
can find an element $y$ in $\mathfrak{v}$ such that

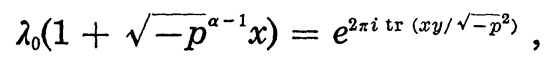

for $x \in \mathfrak{D}$. Then we see

$$
\begin{aligned}
\sum_{a \in \bmod \left(\sqrt{-p^{\alpha}}\right)} \lambda_{0} \eta_{0}(a) e^{2 \pi i \operatorname{tr}(a / b)}= & \sum_{a \in \mathcal{O} \bmod \left(\sqrt{-p^{\alpha-1}}\right)} \lambda_{0} \eta_{0}(a) e^{2 \pi i \operatorname{tr}(a / b)} \\
& \times \sum_{x \in \bmod (\sqrt{-p})} \lambda_{0}\left(1+\sqrt{-p^{\alpha-1}} x\right) e^{2 \pi i \operatorname{tr}\left(a x / \sqrt{-p^{2}}\right)} \\
= & p \sum_{\substack{a \in \mathrm{mod}\left(\sqrt{-p^{\alpha-1}}\right) \\
a+y \equiv 0 \operatorname{mdd}(\sqrt{-p})}} \lambda_{0} \eta_{0}(a) e^{2 \pi i \operatorname{tr}(a / b)} \\
= & \eta_{0}(-y) \sum_{a \in \mathbb{\operatorname { m o d }}\left(\sqrt{-p^{\alpha}}\right)} \lambda_{0}(a) e^{2 \pi i \operatorname{tr}(a / b)} .
\end{aligned}
$$

Hence we obtain

$$
\mathfrak{g}(\lambda \eta)=(b /|b|)^{x^{\prime}-1} \eta_{0}(-y) g(\lambda) .
$$

If we note 


$$
N\left(1+\sqrt{-p^{\alpha-1}} x\right) \equiv 1\left(\bmod p^{[\alpha / 2]}\right),
$$

we see the above argument also gives

$$
g(\lambda \eta \chi \circ N)=(b /|b|)^{\kappa^{\prime}-1} \eta_{0}(-y) g(\lambda \chi \circ N) .
$$

From (4.2) and (4.3), we obtain $\mathfrak{g}(\lambda \eta \chi \circ N) / \mathfrak{g}(\lambda \chi \circ N)=\mathfrak{g}(\lambda \eta) / \mathfrak{g}(\eta)$. This completes the proof.

Let $P=p^{\nu}$, and $\psi$ a character modulo $P$ such that $v_{p}\left(f_{\psi}\right) \leq[\nu / 2]$. For a primitive form $\theta_{\lambda}$ in $S_{\kappa}^{0}(P, \psi)$ associated with Grössencharacter $\lambda$ of $\boldsymbol{Q}(\sqrt{-p})$, put

$$
S\left(\theta_{\lambda}\right)=\left\{f \in S_{\kappa}^{0}(P, \psi)|f| \tilde{U}_{\chi}=c_{\lambda}(\chi) f \quad \text { for } \chi \in X\left(p^{[(\nu-1) / 2]}\right)\right\}
$$

where $\theta_{\lambda} \mid \tilde{U}_{\chi}=c_{\lambda}(\chi) \theta_{\lambda}$. Then the above proposition shows that if $\kappa \geq 2$, we can find a Grössencharacter $\eta$ and a modular form $g$ such that $F(z)=$ $g\left(p^{[(\nu-1 / 2]} z\right) \theta_{\eta}(z)$ belongs to $S\left(\theta_{\lambda}\right)$.

Now we return to our example. In the above notation we have

$$
S\left(\theta_{\text {III }}\right)=S_{2}^{0}(11,4,1) \oplus S_{2}^{0}(11,4,-1) \text {. }
$$

We can choose primitive forms $f \in S_{2}^{0}(11,4,1)$ and $g^{i} \in S^{0}(11,4,-1), 1 \leq i \leq 3$, so that $\theta_{\mathrm{I}}, \theta_{\mathrm{III}}, f, f \mid R_{e}, g^{i}$, and $g^{i} \mid R_{s}(1 \leq i \leq 3)$ form a basis of $S\left(\theta_{\mathrm{III}}\right)$, where $\varepsilon$ is the quadratic residue symbol as before. Let $\omega$ be a character modulo 11 such that $\omega(-1)=-1$, and $E_{\bar{\omega}}(z)$ the Eisenstein series in $M_{1}(11, \bar{\omega})$, that is,

$$
E_{\bar{\omega}}(z)=-\frac{L(0, \bar{\omega})}{2}+\sum_{n=1}^{\infty} \sum_{d\lfloor n} \bar{\omega}(d) e^{2 \pi i n z}
$$

Then we can find a uniquely determined Grössencharacter of $\boldsymbol{Q}(\sqrt{-11})$ modulo $\left(\sqrt{-11^{2}}\right)$ which satisfies $\theta_{\eta} \in S_{1}\left(11^{3}, \omega\right)$ and $F(z)=E_{\bar{\omega}}(p z) \theta_{\eta}(z) \in S\left(\theta_{\text {III }}\right)$. By noting $F(z) \mid R_{s}=F(z)$, we see $F(z)$ can be expressed as follows;

$$
F(z)=a \theta_{\mathrm{I}}+b \theta_{\mathrm{III}}+c\left(f+f \mid R_{\mathrm{s}}\right)+\sum_{i=1}^{3} d_{i}\left(g^{i}+g^{i} \mid R_{s}\right) .
$$

Let $K$ be the field generated by all the Fourier coefficients of $F(z), \theta_{\mathrm{I}}, \theta_{\mathrm{III}}$, $f$, and $g^{i}$, then $a, b, c$, and $d_{i}$ are contained in $K$. Assume $a \neq 0$, and let $\mathfrak{p}$ be a prime ideal of $K$ which divides the denominator of $a$. If we can verify that $b / a, c / a$, and $d_{i} / a$ are $p$-integral and $b / a \equiv 0, c / a \equiv 0(\bmod \mathfrak{p})$, then by Deligne and Serre [2, Lemma 6.11], we can find a primitive form $g$ in $\left\{g^{i}, g^{i} \mid R_{\varepsilon}\right\}$ such that 


$$
\theta_{\mathrm{III}} \equiv g(\bmod \mathfrak{p}) .
$$

Let us check this. First we must calculate $a$. In order to do this, the following Lemma is useful.

LEMMA 4.5. Let $f$, and $g_{i}(1 \leq i \leq n)$ be primitive forms, and $F(z)$ a cusp forms such that

$$
F(z)=\alpha f+\sum_{i=1}^{n} \beta_{i} g_{i} .
$$

Let $a_{n}, b_{n}^{i}$, and $c_{n}$ denote the $n$-th Fourier coefficients of $f, g_{i}$, and $F$ respectively. For a polynomial $T(X)=\sum_{j=1}^{\ell} A_{j} X^{j}$ and a prime $q$, assume $T\left(b_{q}^{i}\right)=0$ for $i, 1 \leq i \leq n$. Then one has

$$
T\left(a_{q}\right) \alpha=\sum_{m=0}^{\ell} \sum_{r=0}^{[m / 2]}\left(\left(\begin{array}{c}
m \\
r
\end{array}\right)-\left(\begin{array}{c}
m \\
r-1
\end{array}\right)\right)\left(p^{x-1}\right)^{r} c_{p^{m-2}} A_{\ell-m}
$$

where $\left(\begin{array}{c}m \\ r\end{array}\right)=m ! / r !(m-r) !$.

This is an easy consequencer of Exercise $3.27^{\prime}$ in [13], and we omit the proof. As $T(X)$, we can take the characteristic polynomial of $T_{q}$ acting on the space spanned by $g_{i}$.

Applying the above Lemma taking $\omega=\varepsilon$, we find $a=0$, and we cannot proceed anymore. In stead of $F(z)$ for $\omega=\varepsilon$, we take the following as $F$;

$$
F^{\prime}(z)=\sum_{\omega} E_{\bar{\omega}}(p z) \theta_{\eta}(z),
$$

where $\omega$ runs through all characters modulo 11 such that $\omega(-1)=-1$ and $\eta$ is the Grössencharacter of $\boldsymbol{Q}(\sqrt{-11})$ such that $\theta_{\eta} \in S_{1}^{0}\left(11^{3}, \omega\right)$. Put

$$
F^{\prime}(z)=a^{\prime} \theta_{\mathrm{I}}+b^{\prime} \theta_{\mathrm{III}}+c^{\prime}\left(f+f \mid R_{\varepsilon}\right)+\sum_{i=1}^{3} d_{i}^{\prime}\left(g^{i}+g^{i} \mid R_{\varepsilon}\right)
$$

as before. Then we find

$$
\begin{gathered}
a^{\prime}=(5 / 22)\left(200 \alpha^{4}+314 \alpha^{3}-612 \alpha^{2}-856 \alpha+54\right) /\left(262 \alpha^{4}+368 \alpha^{3}\right. \\
\left.-895 \alpha^{2}-1003 \alpha+353\right) \\
N\left(200 \alpha^{4}+314 \alpha^{3}-612 \alpha^{2}-856 \alpha+54\right)=2^{5} \cdot 11^{4} \cdot 23 \cdot 197 \\
N\left(262 \alpha^{4}+368 \alpha^{3}-895 \alpha^{2}-1003 \alpha+353\right)=11^{4} \cdot 23 \cdot 99527 .
\end{gathered}
$$

Let $\mathfrak{p}$ be a prime ideal of $K$ which divides $\left(262 \alpha^{4}+368 \alpha^{3}-895 \alpha^{2}-1003 \alpha\right.$ $+353)$ and 99527. We note the Fourier coefficients of $22 F^{\prime}(z)$ are integral. By means of Lemma 4.5 and some calculation, we can check the condition 
on $a^{\prime}, b^{\prime}, c^{\prime}$, and $d_{i}^{\prime}$ mentioned before. For example, the assertion that $d_{i}^{\prime} / a^{\prime}$ is $p$-integral can be verified in the following way. Let $a_{n}, b_{n}, f_{T_{n}}(X)$, and $g_{T_{n}}(X)$ be as in the table. Let $q$ be a prime such that $\varepsilon(q)=1$, then $g_{T_{q}}(X)$ (resp. $f_{T_{q}}(X)$ ) is of the form $g_{q}(X)^{2}$ (resp. $\left(X-c_{q}\right)^{2}$ ), where $g_{q}(X)$ is a polynomial of degree 3 . To prove $d_{i}^{\prime} / a^{\prime}$ is $\mathfrak{p}$-integral, it is enough to show $g_{q}\left(a_{q}\right)$ and $g_{q}\left(c_{q}\right)$ are prime to $\mathfrak{p}$ and $g_{q}(X) \equiv 0 \bmod \mathfrak{p}$ does not have mutiple roots for a prime $q$ with $\varepsilon(q)=1$. We take $q=3$. Then we have

$$
\begin{array}{ll}
g_{3}\left(a_{3}\right)=-6 \alpha^{4}-2 \alpha^{3}+24 \alpha^{2}+6 \alpha-18, & N\left(g_{3}\left(a_{3}\right)\right)=2^{5} \cdot 11 \\
g_{3}\left(c_{3}\right)=4 \alpha^{4}+6 \alpha^{3}-8 \alpha^{2}-14 \alpha-8, & N\left(g_{3}\left(c_{3}\right)\right)=2^{5} \cdot 11^{2} .
\end{array}
$$

Hence $g_{3}\left(a_{3}\right)$ and $g_{3}\left(c_{3}\right)$ are prime to $\mathfrak{p}$. The second condition can be checked easily, since we know one $\operatorname{root} b_{3}$ of $g_{3}(X) \equiv 0(\bmod p)$. We omit the details. Thus we obtain

Proposition 4.6. Let $\theta_{\mathrm{III}} \in S_{2}(11,4,-1)$ and $S_{\mathrm{III}}^{0}\left(\subset S_{2}(11,4,-1)\right)$ be as before. Let $K$ be the field generated by the Fourier coefficients of $\theta_{\mathrm{III}}$ and the primitive forms in $S_{\mathrm{III}}^{0}$, and $\mathfrak{p}$ be a prime ideal of $K$ which divides $262 \alpha^{4}+368 \alpha^{3}-895 \alpha^{2}-1003 \alpha+353$ and 99527. Then there exists a primitive form $g$ in $S_{\text {III }}^{0}$ which satisfies

$$
\theta_{\text {III }} \equiv g(\bmod \mathfrak{p}) .
$$

Now the coefficient $a$ in (4.4) can be written as follows;

$$
a=\frac{\left\langle\theta_{\mathrm{III}}, F(z)\right\rangle}{\left\langle\theta_{\mathrm{III}}, \theta_{\mathrm{III}}\right\rangle},
$$

where $\langle$,$\rangle denotes the Petersson inner product, and the coefficient a^{\prime}$ in (4.5) can be expressed as a sum of such numbers. By means of a result. of Shimura [16], we can relate the number $a$ to the special values of zeta functions. We introduce some notations. For positive integer $N, \kappa$ and a Dirichlet character $\omega$ modulo $N$ such that $\omega(-1)=(-1)^{x}$, put

$$
E_{\kappa, N}^{*}(z, s, \omega)=\sum_{r \in \Gamma_{\infty} \backslash \Gamma_{0}(N)} \omega(d)(c z+d)^{-\kappa}|c z+d|^{-2 s}, \quad \gamma=\left(\begin{array}{ll}
a & b \\
c & d
\end{array}\right),
$$

where $\Gamma_{\infty}=\left\{\left(\begin{array}{ll}1 & b \\ 0 & 1\end{array}\right) \mid b \in Z\right\}$, and

$$
E_{\kappa, N}(z, s, \omega)=\sum_{m, n} \omega(n)(m N z+n)^{-\kappa}|m N z+n|^{-2 s},
$$

where the summation is taken over all $(m, n) \in Z^{2}, \neq 0$. These are abso- 
lutely convergent for $\operatorname{Re}(2 s)>2-\kappa$, and we have

$$
E_{\kappa, N}(z, s, \omega)=2 L_{N}(2 s+\kappa, \omega) E_{\kappa, N}^{*}(z, s, \omega),
$$

where $L_{N}(s, \omega)=\sum_{(N, n)=1} \omega(n) n^{-s}$. For $\kappa>0$, we put

$$
E_{\kappa, N}(z, \omega)=E_{\kappa, N}(z, 0, \omega), \quad E_{\kappa, N}^{*}(z, \omega)=E_{\kappa, N}^{*}(z, 0, \omega) .
$$

If $\kappa \neq 2$, or $\omega$ is not trivial, $E_{\kappa, N}(z, \omega)$ and $E_{k, N}(z, \omega)$ belongs to $G_{k}(N, \bar{\omega})$.

Proposition 4.7. For a prime $p \equiv 3(\bmod 4)$, let $\omega$ be $a$ character modulo $p$ and $\theta_{2}$ (resp. $\theta_{\eta}$ ) a primitive form associated with a Grössencharacter $\lambda($ resp. $\eta)$ of $Q(\sqrt{-p})$ belonging to $S_{k}^{0}(P, \psi)$ (resp. $S_{\kappa^{\prime}}^{0}(P, \psi \omega)$ ) for $P=p^{\nu}$ and a character $\psi$ which satisfy $v_{p}\left(\mathfrak{F}_{\psi}\right) \leq \nu / 3$. Assume that $\kappa>\kappa^{\prime}$ and that $\kappa-\kappa^{\prime} \neq 2$ or $\omega$ is not trivial. Put $F(z)=E_{\kappa-\kappa^{\prime}},\left(p^{[(\nu-1) / 2]} z, \omega\right) \theta_{\eta}(z)$. If $F(z)$ belongs to $S\left(\theta_{\lambda}\right)$, then

$$
\frac{\left\langle\theta_{\lambda}, F\right\rangle}{\left\langle\theta_{\lambda}, \theta_{\lambda}\right\rangle}=\frac{4(\kappa-1) \pi^{2}}{p^{\nu-[(\nu-1) / 2]} L(1, \varepsilon)} \frac{L\left(\left(\kappa-\kappa^{\prime}\right) / 2, \lambda^{\prime} \eta\right) L\left(\left(\kappa-\kappa^{\prime}\right) / 2, \lambda^{\prime} \eta^{\prime-1}\right)}{L\left(1, \lambda^{\prime} \lambda\right)},
$$

where $\lambda^{\prime}(\mathfrak{a})=\overline{\lambda(\overline{\mathfrak{a}})}, \eta^{\prime}(\mathfrak{a})=\overline{\eta(\overline{\mathfrak{a}})}$ for an ideal $\mathfrak{a}$ in $\boldsymbol{Q}(\sqrt{-p})$.

Proof. Let $\Phi$ denote a fundamental domain of $\mathfrak{S}$ with respect to $\Gamma_{0}(P)$. Put $\mu=[(\nu-1) / 2]$. Let $\Gamma$ be a subgroup of $\Gamma_{0}(P)$ given by

$$
\Gamma=\left\{\left(\begin{array}{ll}
a & b \\
c & d
\end{array}\right) \in \Gamma_{0}(P) \mid a \equiv d \equiv 1\left(\bmod p^{\mu}\right)\right\},
$$

and $\Phi^{\prime}$ a fundamental domain for $\Gamma$. We note $\Gamma$ is a normal subgroup of $\Gamma_{0}\left(p^{\mu+1}\right)$. Let $\left\{a_{j}\right\}$ be a complete system of representatives of $Z$ modulo $p^{\mu}$, then $\Gamma_{0}\left(p^{\mu+1}\right)=\bigcup_{j} \Gamma_{0}(P) \alpha_{j}$ is a disjoint union, where $\alpha_{j}=\left(\begin{array}{cc}1 & 0 \\ p^{\mu+1} a_{j} & 1\end{array}\right)$. For the sake of simplicity, we put

$$
E(z, s)=E_{x-k^{\prime}, p}(z, s, \omega), E(z, s)^{*}=E_{x-k^{\prime}, P}^{*}(z, s, \omega) .
$$

We note $E_{\kappa-\kappa^{\prime}, p^{\mu+1}}(z, s, \omega)=E_{\kappa-\kappa^{\prime}, p}\left(p^{\mu} z, s, \omega\right)$, and

$$
E_{\kappa-\kappa^{\prime}, p^{\mu+1}}^{*}(z, s, \omega)=\sum_{j} E(z, s)^{*} \mid\left[\alpha_{j}\right],
$$

where $E(z, s)^{*}\left|[\gamma]=\omega(d)(c z+d)^{-\left(x-k^{\prime}\right)}\right| c z+\left.d\right|^{-2 s} E(\gamma(z), s)^{*}$ for $\gamma=\left(\begin{array}{ll}a & b \\ c & d\end{array}\right)$ $\in S L_{2}(Z)$. We have

$$
\begin{aligned}
I & =\int_{\Phi} \bar{\theta}_{\lambda} \theta_{\eta} E_{\kappa-k^{\prime}, p}\left(p^{\mu} z, s, \omega\right) y^{s+\kappa-2} d x d y \\
& =c(s) \sum_{j} \int_{\Phi^{\prime}} \bar{\theta}_{\lambda} \theta_{\eta}\left(E(z, s)^{*} \mid\left[\alpha_{j}\right]\right) y^{s+\kappa-2} d x d y,
\end{aligned}
$$


where $c(s)=2 L_{P}\left(2 s+\kappa-\kappa^{\prime}, \omega\right) /\left[\Gamma_{0}(P): \Gamma\right]$. If $a_{j} \equiv 0\left(\bmod p^{\mu}\right)$, then for $\operatorname{Re}(2 s)>2-\left(\kappa-\kappa^{\prime}\right)$ as in $\S 2$ of [16]

$$
\begin{aligned}
\int_{\Phi^{\prime}} \bar{\theta}_{\lambda} \theta_{\eta} & \left(E(z, s)^{*} \mid\left[\alpha_{j}\right]\right) y^{s+\kappa-2} d x d y \\
& =\left[\Gamma_{0}(P): \Gamma\right] \int_{\Phi} \bar{\theta}_{\lambda} \theta_{\eta} E(z, s)^{*} y^{s+\kappa-2} d x d y \\
& =\left[\Gamma_{0}(P): \Gamma\right](4 \pi)^{-(s+\kappa-1)} \Gamma(s+\kappa-1) D\left(s+\kappa-1, \theta_{\lambda^{\prime}}, \theta_{\eta}\right),
\end{aligned}
$$

where $D(s, f, g)=\sum_{n=1}^{\infty} a_{n} b_{n} n^{-s}$ for $f(z)=\sum_{n=1}^{\infty} a_{n} e^{2 \pi i n z}$ and $g(z)=\sum_{n=1}^{\infty} b_{n} e^{2 \pi i n z}$. $\lambda^{\prime}$ is the Grössencharacter given by $\lambda^{\prime}(\mathfrak{a})=\overline{\lambda(\bar{a})}$. If $a_{j} \neq 0\left(\bmod p^{\mu}\right)$, then put $a_{j}=v p^{\nu-\mu-1-\tau}$ with a positive integer $\tau$ and $v$ prime to $p$. If we define $\beta_{v}$ by

$$
\beta_{v}=\left(\begin{array}{cc}
1 & v / p^{\tau} \\
0 & 1
\end{array}\right)
$$

then $\alpha_{j}^{-1}=\eta_{P}^{-1} \beta_{v} \eta_{P}$. Since $\alpha_{j} \in \Gamma_{o}\left(p^{\mu+1}\right)$ and $\Gamma$ is a normal subgroup of $\Gamma_{0}\left(p^{\mu+1}\right)$, we see

$$
\begin{aligned}
\int_{\Phi^{\prime}} \bar{\theta}_{\lambda} \theta_{\eta} & \left(E(z, s)^{*} \mid\left[\alpha_{j}\right]\right) y^{s+\varepsilon-2} d x d y \\
& =\int_{\Phi^{\prime}}\left(\overline{\theta_{\lambda} \mid\left[\alpha_{j}^{-1}\right]_{\kappa}}\right)\left(\theta_{\eta} \mid\left[\alpha_{j}^{-1}\right]_{\kappa^{\prime}}\right) E(z, s)^{*} y^{s+\kappa-2} d x d y \\
& =\int_{\Phi^{\prime}}\left(\overline{\theta_{\lambda} \mid W_{P}^{-1}\left[\beta_{v}\right]_{\kappa}}\right)\left(\theta_{\eta} \mid W_{P}^{-1}\left[\beta_{v}\right]_{\kappa^{\prime}}\right) E(z, s)^{*} \mid W_{P}^{-1} y^{s+\kappa-2} d x d y,
\end{aligned}
$$

where $\left.E(z, s)^{*}\left|W_{P}^{-1}=E\left(\eta_{P}^{-1}(z), s\right)^{*}\left(-p^{y / 2} z\right)^{-\left(\kappa-k^{\prime}\right)}\right| p^{y / 2} z\right|^{-2 s}$. Now we have

LEMMA 4.8. For a character $\psi$ modulo $p^{\nu-1}$, let $f$ be a primitive form in $S_{k}^{0}(P, \psi)$ for $P=p^{\nu}$. For a character $\chi$, put $f_{x}=f \mid R_{\chi}$. If $\nu \geq 2$, for $\beta_{v}$ $=\left(\begin{array}{cc}1 & v / p^{\sigma} \\ 0 & 1\end{array}\right)$ with $\tau \geq 1$ and $(v, p)=1$, it holds

$$
f \mid\left[\beta_{v}\right]_{\kappa}= \begin{cases}\frac{1}{p-1} \sum_{\chi} \chi(v) g(\bar{\chi}) f_{x} & \text { if } \tau=1 \\ \frac{1}{p^{\tau}(1-1 / p)} \sum_{\chi} \chi(v) g(\bar{\chi}) f_{x} & \text { otherwise }\end{cases}
$$

where $\chi$ runs through all characters modulo $p$ if $\tau=1$ and all characters with the conductor $p^{\tau}$ if $\tau \geq 2$. For the trivial character $\chi_{1}$, we put $g\left(\chi_{1}\right)$ $=-1$.

Proof. By the definition of the twisting operator, we have 


$$
g(\bar{\chi}) f_{\chi}=\sum_{\substack{u \bmod p^{\sigma} \\(u, p)=1}} \bar{\chi}(u) f \mid\left[\alpha_{u}\right]_{\kappa},
$$

where $\tilde{\mathrm{f}}_{x}=p^{\sigma}$ and $\alpha_{u}=\left(\begin{array}{cc}1 & u / p^{\sigma} \\ 0 & 1\end{array}\right)$. If $\tau=1$, we see

$$
\begin{aligned}
\sum_{f_{\chi} \leq p} \chi(v) g(\bar{\chi}) f_{\chi} & =\sum_{f_{\chi}=p} \chi(v) \sum_{(u, p)=\tau} \bar{\chi}(u) f \mid\left[\alpha_{u}\right]_{\kappa}-f \\
& =\sum_{\substack{\mathfrak{f}_{\chi} \leq p \\
(u, p)=1}} \chi(v) \bar{\chi}(u) f \mid\left[\alpha_{u}\right]_{\kappa} \\
& =(p-1) f \mid\left[\alpha_{v}\right]_{\kappa} .
\end{aligned}
$$

This prove the case where $\tau=1$. We can treat the case where $\tau \geq 2$ in the same way, because for $\chi^{\prime}$ with, $\mathfrak{f}_{\chi^{\prime}} \leq p^{\sigma-1}$, we have

$$
\sum_{\substack{v \bmod \\(v, p)=1}} \chi^{\prime}(v) f \mid\left[\alpha_{v}\right]_{\kappa}=0
$$

and we omit the details.

Put $f=\theta_{\lambda}\left|W_{P}^{-1}, g=\theta_{\eta}\right| W_{P}^{-1}, \quad$ and $\quad E^{\prime}(z, s)=E(z, s)^{*} \mid W_{P}^{-1}$.

For $\beta_{v}$ with $\tau=1$, we have

$$
\begin{aligned}
I_{1}= & \sum_{\substack{v \text { mod } p \\
(v, p)=1}} \int_{\Phi^{\prime}}\left(\overline{f \mid\left[\beta_{v}\right]_{\kappa}}\right)\left(g \mid\left[\beta_{v}\right]_{\kappa^{\prime}}\right) E^{\prime}(z, s) y^{s+\varepsilon-2} d x d y \\
= & \frac{1}{(p-1)^{2}} \int_{\Phi^{\prime}} \sum_{v}\left(\sum_{\chi} \overline{\chi(v) g(\bar{\chi}) f_{\chi}}\right)\left(\sum_{\chi^{\prime}} \chi^{\prime}(v) \mathfrak{g}\left(\bar{\chi}^{\prime}\right) g_{\chi^{\prime}}\right) \\
& \times E^{\prime}(z, s) y^{s+\kappa-2} d x d y \\
= & \frac{1}{(p-1)} \int_{\Phi^{\prime}} \sum_{\chi} \overline{\mathfrak{g}(\bar{\chi})}(\bar{\chi}) \bar{f}_{\chi} g_{\chi} E^{\prime}(z, s) y^{s+\varepsilon-2} d x d y .
\end{aligned}
$$

We have by Prop. 3.5

$$
\begin{aligned}
\left.\overline{\left(f_{\chi} \mid W_{P}\right.}\right)\left(g_{\chi} \mid W_{P}\right) & =\left(\overline{\theta_{\lambda} \mid W_{P}^{-1} R_{\chi} W_{P}}\right)\left(\theta_{\mu} \mid W_{P}^{-1} R_{\chi} W_{P}\right) \\
& \left.=\overline{\left(\theta_{\lambda} \mid \tilde{U}_{\chi} R_{\bar{\chi}}\right.}\right)\left(\theta_{\mu} \mid \tilde{U}_{\chi} R_{\bar{\chi}}\right) \\
& =\left(\overline{\theta_{\lambda} \mid R_{\bar{\chi}}}\right)\left(\theta_{\mu} \mid R_{\bar{\chi}}\right),
\end{aligned}
$$

since $F(z) \in S\left(\theta_{\lambda}\right)$. Hence we obtain

$$
\begin{aligned}
I_{1} & \left.=\frac{1}{(p-1)} \sum_{\bar{x}} \overline{g(\bar{\chi})} g(\bar{\chi}) \int_{\mathscr{\Phi}^{\prime}} \overline{\left(f_{\chi} \mid W_{P}\right.}\right)\left(g_{x} \mid W_{P}\right) E(z, s)^{*} y^{s+\kappa-2} d x d y \\
& =\frac{1}{(p-1)} \sum \overline{g(\bar{\chi})} g(\bar{\chi}) \int_{\Phi^{\prime}}\left(\overline{\theta_{\lambda} \mid R_{\bar{\chi}}}\right)\left(\theta_{\eta} \mid R_{\bar{\chi}}\right) E(z, s)^{*} y^{s+\kappa-2} d x d y \\
& =(p-1)\left[\Gamma_{0}(P): \Gamma\right](4 \pi)^{-(s+\kappa-1)} \Gamma(s+\kappa-1) D\left(s+\kappa-1 ; \theta_{\lambda^{\prime}}, \theta_{\eta}\right) .
\end{aligned}
$$


For $\beta_{v}=\left(\begin{array}{cc}1 & v / p^{\tau} \\ 0 & 1\end{array}\right)$ with $\tau \geq 2$, we can show in the same way

$$
\begin{aligned}
\left.\sum_{\substack{v \bmod p \\
(v, p)=1}} \int_{\Phi^{\prime}} \overline{\left(f \mid\left[\beta_{v}\right]_{\kappa}\right.}\right)\left(g \mid\left[\beta_{v}\right]_{\kappa^{\prime}}\right) E^{\prime}(z, s) y^{s+\kappa-2} d x d y & \\
= & \frac{1}{(p-1)}\left(p^{\tau}-2 p^{\tau-1}+p^{\tau-2}\right)(4 \pi)^{-(s+\kappa-1)} \Gamma(s+\kappa-1) \\
& D\left(s+\kappa-1, \theta_{\lambda^{\prime}}, \theta_{\eta}\right) .
\end{aligned}
$$

By (4.6), (4.7), (4.8), and (4.9), we obtain

$$
I=2 L_{P}\left(2 s+\kappa-\kappa^{\prime}, \omega\right) p^{\mu}(4 \pi)^{-(s+\kappa-1)} \Gamma(s+\kappa-1) D\left(s+\kappa-1, \theta_{\lambda^{\prime}}, \theta_{\eta}\right) .
$$

By Lemma 1 of [16], this is equal to

$$
2 p^{\mu}(4 \pi)^{-(s+\kappa-1)} \Gamma(s+\kappa-1) L\left(s+\frac{\kappa-\kappa^{\prime}}{2}, \lambda^{\prime} \eta\right) L\left(s+\frac{\kappa-\kappa^{\prime}}{2}, \lambda^{\prime} \eta^{\prime-1}\right),
$$

where $\eta^{\prime}(\mathfrak{a})=\overline{\eta(\overline{\mathfrak{a}})}$ for ideals $\mathfrak{a}$ in $\boldsymbol{Q}(\sqrt{-p})$. Putting $s=0$, we obtain

$$
\left\langle\theta_{\lambda}, F(z)\right\rangle=2 p^{\mu}(4 \pi)^{-(\kappa-1)} \Gamma(\kappa-1) L\left(\frac{\kappa-\kappa^{\prime}}{2}, \lambda^{\prime} \eta\right) L\left(\frac{\kappa-\kappa^{\prime}}{2}, \lambda^{\prime} \eta^{\prime-1}\right) .
$$

On the other hand, by (2.5) in [14], we have

$$
\left\langle\theta_{\lambda}, \theta_{\lambda}\right\rangle=(4 \pi)^{-\varepsilon} \Gamma(\kappa) \frac{\pi}{3} P(1+1 / p) \operatorname{Res}_{s=\varsigma} D\left(s, \theta_{\lambda^{\prime}}, \theta_{\lambda}\right) .
$$

As above, we have

$$
D\left(s, \theta_{\lambda^{\prime}}, \theta_{\lambda}\right)=\frac{L\left(s-\kappa+1, \lambda^{\prime} \lambda\right) L\left(s-\kappa+1, \lambda_{1}\right)}{L_{P}\left(2 s-2 \kappa+2, \chi_{1}\right)},
$$

where $\chi_{1}$ is the trivial character and $\lambda_{1}(\mathfrak{a})=1$ if $\mathfrak{a}$ is prime to $p$ and $\lambda_{1}(\mathfrak{a})$ $=0$ otherwise. Hence we obtain

$$
\left\langle\theta_{\lambda}, \theta_{\lambda}\right\rangle=(4 \pi)^{-(\kappa-1)} \Gamma(\kappa)\left(2 \pi^{2}\right)^{-1} P L\left(1, \lambda^{\prime} \lambda\right) L(1, \varepsilon),
$$

and thus

$$
\frac{\left\langle\theta_{\lambda}, F\right\rangle}{\left\langle\theta_{\lambda}, \theta_{\lambda}\right\rangle}=\frac{4(\kappa-1) \pi^{2}}{p^{\nu-\mu} L(1, \varepsilon)} \frac{L\left(\left(\kappa-\kappa^{\prime}\right) / 2, \lambda^{\prime} \eta\right) L\left(\left(\kappa-\kappa^{\prime}\right) / 2, \lambda^{\prime} \eta^{\prime-1}\right)}{L\left(1, \lambda^{\prime} \lambda\right)} .
$$

This completes the proof.

\section{Appendix}

I. Let $N=13^{3}, \kappa=2$, and $\psi=$ the trivial character. Then we find 
$\operatorname{dim} S_{2}\left(13^{3}, 4,1\right)=6$, and $\operatorname{dim} S_{2}\left(13^{3}, 4,-1\right)=8$. Let $f_{T_{n}}(X)$ and $g_{T_{n}}(X)$ denote the characteristic polynomial of $T_{n}$ on the spaces $S_{2}\left(13^{3}, 4,1\right)$ and $S_{2}\left(13^{3}\right.$, $4,-1)$ respectively. Then for $n=2$ and $3, f_{T_{n}}(X)$ and $g_{T_{n}}(X)$ are given by

\begin{tabular}{c|c}
$n$ & $f_{T_{n}}(X)$ \\
\hline 2 & $X^{6}-\left(-\alpha^{3}+3 \alpha+8\right) X^{4}+\left(\alpha^{5}-\alpha^{4}-9 \alpha^{3}+3 \alpha^{2}+17 \alpha+15\right) X^{2}$ \\
3 & $\left(X^{3}-(-2) X^{2}+\left(\alpha^{2}-5\right) X-\left(\alpha^{3}-\alpha^{2}-4 \alpha+5\right)\right)^{2}$
\end{tabular}

\begin{tabular}{c|c}
$n$ & $g_{T_{n}}(X)$ \\
\hline 2 & $X^{8}-\left(\alpha^{3}-3 \alpha+13\right) X^{6}+\left(-3 \alpha^{5}-\alpha^{4}+24 \alpha^{3}+3 \alpha^{2}-42 \alpha+51\right) X^{4}$ \\
& $-\left(-18 \alpha^{5}+108 \alpha^{3}-8 \alpha^{2}-145 \alpha+80\right) X^{2}$ \\
& $+\left(-17 \alpha^{5}-\alpha^{4}+91 \alpha^{3}-9 \alpha^{2}-108 \alpha+41\right)$ \\
& $\left(X^{4}-2 X^{3}+\left(-\alpha^{2}-5\right) X^{2}-\left(-2 \alpha^{5}-2 \alpha^{4}+9 \alpha^{3}+5 \alpha^{2}-8 \alpha-9\right) X\right.$ \\
& $\left.+\left(-4 \alpha^{5}-2 \alpha^{4}+16 \alpha^{3}+8 \alpha^{2}-10 \alpha-2\right)\right)^{2}$
\end{tabular}

where $\alpha=e^{2 \pi i / 13}+e^{-\pi i / 13}$. We remark the following. Let $N$ denote the norm from $\boldsymbol{Q}(\alpha)$ to $\boldsymbol{Q}$, then

$$
N\left(f_{T_{2}}(0)\right)=443, \quad N\left(g_{T_{2}}(0)\right)=53 \cdot 79 .
$$

On the other hand, let $\varepsilon_{0}=(3+\sqrt{13}) / 2$ be a fundamental unit of $Q(\sqrt{13})$, then

$$
N_{Q(\sqrt{13}) / Q}\left(\varepsilon_{0}^{13}-1\right)=-3 \cdot 53 \cdot 79 \cdot 443 \text {. }
$$

Such a relation has been noticed in [3, Remark 2.1.] for the case $N=5^{3}$.

II. Let $N=19^{3}, \kappa=2$, and $\psi$ the trivial character. Then we find $\operatorname{dim} S_{2}\left(19^{3}, 4,1\right)=12$ and $\operatorname{dim} S_{2}\left(19^{3}, 4,-1\right)=16$. Let $\theta_{\mathrm{I}}(z)=\sum a_{n} e^{2 \pi i n z} \in S_{2}\left(19^{3}\right.$, 4,1) (resp. $\theta_{\mathrm{III}}(z)=\sum b_{n} e^{2 \pi i n z} \in S_{2}(19,4,-1)$ ) be a primitive form associated with a Grössencharacter of $Q(\sqrt{-19})$ and $S_{\mathrm{I}}^{0}\left(\mathrm{resp}\right.$. $\left.S_{\mathrm{III}}^{0}\right)$ the orthogonal complement of the space spanned by $\theta_{\mathrm{I}}$ (resp. $\theta_{\mathrm{III}}$ ). We denote by $f_{T_{n}}(X)$ (resp. $\left.g_{T_{n}}(X)\right)$ the characteristic polynomial of $T_{n}$ acting on $S_{\mathrm{I}}^{0}$ (resp. $S_{\mathrm{III}}^{0}$ ). Let $\alpha$ $=e^{2 \pi i / 19}+e^{-2 \pi i / 19}$ and let $\left(x_{1}, x_{2}, \cdots, x_{9}\right)$ denote the number $\sum_{i=1}^{9} x_{i} \alpha^{9-i}$ in $\boldsymbol{Q}(\alpha)$. Then we have

In the preparation of the tables in the Appendix, we used FACOM M190 at Data Processing center of Kyoto University. 


$$
\begin{aligned}
f_{T_{2}}(X)=X^{12} & -A_{10} X^{10}+A_{8} X^{8}-A_{6} X^{6}+A_{4} X^{4}-A_{2} X^{2}+A_{0} \\
A_{10} & =(0,0,0,0,0,0,0,0,18) \\
A_{8} & =(0,3,0,-21,0,42,0,-21,120) \\
A_{6} & =(0,30,-3,-210,17,419,-24,-209,373) \\
A_{4} & =(-2,94,-4,-655,76,1298,-136,-651,558) \\
A_{2} & =(-18,99,103,-687,-124,1356,-50,-711,351) \\
A_{0} & =(-21,26,145,-176,-291,336,163,-187,44)
\end{aligned}
$$

$$
a_{2}=0, N\left(f_{T_{2}}\left(a_{2}\right)\right)=37^{2} \cdot 56536856647
$$

$$
\begin{aligned}
f_{T_{5}}(X)= & \left(X^{6}-A_{5}^{\prime} X^{5}+A_{4}^{\prime} X^{4}-A_{3}^{\prime} X^{3}+A_{2}^{\prime} X^{2}-A_{1}^{\prime} X+A_{0}^{\prime}\right)^{2} \\
& A_{5}^{\prime}=(0,0,0,0,1,1,-4,-3,-1) \\
& A_{4}^{\prime}=(0,1,0,-7,-2,11,9,3,-15) \\
& A_{3}^{\prime}=(-4,-4,32,27,-91,-61,105,50,-2) \\
& A_{2}^{\prime}=(4,-5,-26,32,59,-31,-73,-60,38) \\
& A_{1}^{\prime}=(13,2,-119,-10,354,18,-356,-22,47) \\
& A_{0}^{\prime}=(16,18,-113,-105,233,141,-125,19,10)
\end{aligned}
$$

$$
a_{5}=(0,1,0,-7,-1,13,5,-4,-5)
$$$$
N\left(f_{T_{5}}\left(a_{5}\right)\right)=-37 \cdot 227 \cdot 150707 \cdot 56536856647
$$

$$
\begin{aligned}
g_{T_{2}}(X)=X^{16}-B_{14} X^{14}+B_{12} X^{12}-B_{10} X^{10}+B_{8} X^{8}-B_{6} X^{6} & +B_{4} X^{4}-B_{2} X^{2}+B_{0} \\
& B_{14}=(0,0,0,0,0,0,0,0,27) \\
B_{12} & =(0,-3,0,21,0,-42,0,21,294) \\
& B_{10}=(0,-57,1,399,-7,-799,12,404,1657) \\
& B_{8}=(4,-398,-13,2795,-51,-5639,164,2928,5157) \\
B_{6} & =(32,-1263,-149,8940,-108,-18340,844,9980,8723) \\
B_{4} & =(53,-1847,-254,13227,-255,-27848,1845,16178,7321) \\
B_{2} & =(15,-1076,-67,7756,-325,-16788,1453,10589,2464) \\
B_{0} & =(-24,-110,168,708,-418,-1458,450,1112,194)
\end{aligned}
$$

$a_{2}=0, N\left(g_{T_{2}}\left(a_{2}\right)\right)=2^{9} \cdot 19^{2} \cdot 5736557 \cdot 6463381$

$$
\begin{aligned}
g_{T_{5}}(X)= & \left(X^{8}-B_{7}^{\prime} X^{7}+B_{6}^{\prime} X^{6}-B_{5}^{\prime} X^{5}+B_{4}^{\prime} X^{4}-B_{3}^{\prime} X^{3}+B_{2}^{\prime} X^{2}-B_{1}^{\prime} X+B_{0}^{\prime}\right)^{2} \\
& B_{7}^{\prime}=(0,1,0,-7,0,14,1,-6,2) \\
& B_{6}^{\prime}=(0,3,0,-21,-2,42,8,-17,-19) \\
& B_{5}^{\prime}=(4,-10,-29,67,54,-127,-29,49,-30)
\end{aligned}
$$




$$
\begin{aligned}
& B_{4}^{\prime}=(7,-27,-51,175,126,-335,-143,145,112) \\
& B_{3}^{\prime}=(-35,21,254,-143,-492,246,243,-35,121) \\
& B_{2}^{\prime}=(-56,43,395,-236,-857,383,664,-133,-196) \\
& B_{1}^{\prime}=(44,-13,-313,109,574,-189,-264,0,-98) \\
& B_{0}^{\prime}=(43,-4,-281,6,505,13,-248,-32,13) \\
& b_{5}=(0,-1,0,7,1,-13,-5,3,5) \\
& N\left(g_{T_{5}}\left(a_{5}\right)\right)=571 \cdot 3457.51679 \cdot 28579723 \cdot 5736557 \cdot 6463381 .
\end{aligned}
$$

Here $N$ denotes the norm from $\boldsymbol{Q}(\alpha)$ to $\boldsymbol{Q}$. We remark $N\left(f_{T_{2}}\left(a_{2}\right)\right)$ and $N\left(f_{T_{5}}\left(a_{5}\right)\right)$ (resp. $N\left(g_{T_{2}}\left(a_{2}\right)\right)$ and $\left.N\left(g_{T_{5}}\left(a_{5}\right)\right)\right)$ have a common factor 56536856647 (resp. 5736557.6463381).

\section{REFERENCES}

[1] A. O. L. Atkin and W. Li, Twists of newforms and pseudo-eigenvalues of Woperators, Inv. math. 48 (1978), 221-243.

[2] P. Deligne and J. P. Serre, Formes modulaires de poids 1, Ann. scient. Ec. Norm. Sup. $4^{\mathrm{e}}$ serie 7 (1974), 507-530.

[ 3 ] K. Doi and M. Yamauchi, On the Hecke operators for $\Gamma_{0}(N)$ and class fields over quadratic fields, J. Math. Soc. Japan 25 (1973), 629-643.

[4] K. Doi and M. Ohta, On some congruence between cusp forms for $\Gamma_{0}(N)$, Modular functions of one variable V, Lecture Notes in Math., vol. 601, Springer, 1977.

[5] K. Doi and H. Hida, On a congruence of cusp forms and the special values of their Dirichlet series (to appear).

[6] M. Eichler, Eine Verallgemeinerung der Abelschen Integrale, Math. Z. 67 (1957), 267-298.

[ 7 ] - Quadratische Formen und Modulfunktionen, Acta Arith. 4 (1958), 217-239.

[8] H. Hijikata, Explicit formula of the traces of Hecke operators for $\Gamma_{0}(N)$, J. Math. Soc. Japan 26 (1974), 56-82.

[ 9 ] H. Ishikawa, On the trace formula for Hecke operators, J. Fac. Sci. Univ. Tokyo 21 (1974), 357-376.

[10] H. Saito, On Eichler's trace formula, J. Math. Soc. Japan 24 (1972), 333-340.

[11] H. Saito and M. Yamauchi, Trace formula of certain Hecke operators for $\Gamma_{0}\left(q^{\nu}\right)$ Nagoya Math. J. 76 (1979), 1-33.

[12] H. Shimizu, On traces of Hecke operators, J. Fac. Sci. Univ. Tokyo 10 (1963), 1-19.

[13] G. Shimura, Introduction to the arithmetic theory of automorphic functions, Publ. Math. Soc. Japan, No.11, Iwanami Shoten and Princeton University press, 1971.

[14] - On elliptic curves with complex multiplication as factors of the Jacobians of modular function fields, Nagoya Math. J. 43 (1971), 199-208.

[15] — Class fields over real quadratic fields and Hecke operators, Ann. of Math. 95 (1972), 130-190.

[16] — The special values of the zeta functions associated with cusp forms ,Comm. pure appl. Math. 29 (1978), 333-340.

[17] —, The special values of the zeta functions associated with Hilbert modular 
forms, Duke Math. J. 45 (1978), 637-679.

[18] M. Yamauchi, On the traces of Hecke operators for a normalizer of $\Gamma_{0}(N)$, J. Math. Kyoto Univ. 13 (1973), 403-411.

Department of Mathematics College of General Education Kyoto University 